\title{
Analysis of Polarimeter Data for the 2001-2002 RHIC Run
}

A. Bravar, ${ }^{1}$ G. Bunce, ${ }^{1}$ R.V. Cadman, ${ }^{2}$ H. Huang,,${ }^{1}$ O. Jinnouchi, ${ }^{3}$ K. Krueger, ${ }^{2}$ K. Kurita, ${ }^{3}$ Y. Makdisi, ${ }^{1}$ N. Saito, ${ }^{3}$ H. Spinka, ${ }^{2}$ B. Surrow, ${ }^{1}$

E. Stephenson, ${ }^{4}$ D. Underwood, ${ }^{2}$ J. Wood, ${ }^{5}$ and A. Zelenski ${ }^{1}$

\author{
${ }^{1}$ Brookhaven National Laboratory, Upton, New York \\ ${ }^{2}$ Argonne National Laboratory, Argonne, Illinois \\ ${ }^{3}$ RIKEN, Institute of Physical and Chemical Research, Saitama, Japan \\ ${ }^{4}$ Indiana University Cyclotron Facility, Bloomington, Indiana \\ ${ }^{5}$ University of California, Los Angeles, California
}

Collider-Accelerator Department Brookhaven National Laboratory

Upton, NY 11973 


\title{
Analysis of Polarimeter Data for the 2001-2002 RHIC Run
}

\author{
A. Bravar, ${ }^{1}$ G. Bunce, ${ }^{1}$ R.V. Cadman,${ }^{2}$ H. Huang, ${ }^{1}$ O. Jinnouchi, ${ }^{3}$ \\ K. Krueger, ${ }^{2}$ K. Kurita,${ }^{3}$ Y. Makdisi, ${ }^{1}$ N. Saito,${ }^{3}$ H. Spinka,${ }^{2}$ \\ E. Stephenson, ${ }^{4}$ B. Surrow ${ }^{1}$ D. Underwood,${ }^{2}$ J. Wood, ${ }^{5}$ and A. Zelenski ${ }^{1}$ \\ ${ }^{1}$ Brookhaven National Laboratory, Upton, New York 11973 \\ ${ }^{2}$ Argonne National Laboratory, Argonne, Illinois 60439 \\ ${ }^{3}$ RIKEN, Institute of Physical and Chemical Research, Saitama 351-0198, Japan \\ ${ }^{4}$ Indiana University Cyclotron Facility, Bloomington, Indiana 47408 \\ ${ }^{5}$ University of California, Los Angeles, California 90095
}

(Dated: September 9, 2003)

\begin{abstract}
The results of several studies of the $200-\mathrm{MeV}$ (LINAC), AGS (E880), and RHIC polarimeter data from the polarized proton run in 2001/2002 are presented. Much of this work occurred during or immediately after the run. Some of these analyses have implications for the understanding of the performance of the polarized ion source, AGS, and RHIC with polarized protons.

Some of the conclusions include: a) A recalibration of the $200-\mathrm{MeV}$ polarimeter gives results consistent with the older calibration, but high rates in the $200-\mathrm{MeV}$ polarimeter are still a problem. b) No evidence of sizable systematic effects was found in several tests of the AGS polarimeter with a thin carbon fiber target. c) Significant polarization loss in the AGS was observed above G $\gamma=7.5$, but not between injection and $\mathrm{G} \gamma=7.5$. d) The magnitude of the flattop asymmetry in the RHIC polarimeters decreases with time in a fill. e) The RHIC polarimeter analyzing power on flattop is greater than or equal to that at injection. f) Unexplained systematic effects were observed in the RHIC polarimeter, but the implications for the measured polarization asymmetry are not clear. These effects were not isolated events, but occurred throughout the run. Conceivably the data could indicate that the present RHIC polarimeter design will not be able to achieve a goal of a $\pm 5 \%$ measurement of the beam polarization.
\end{abstract}




\section{INTRODUCTION}

This note documents measurements made with the 200-MeV, AGS or E880, and RHIC Blue and Yellow ring polarimeters during the polarized running in late 2001 and early 2002 (RHIC run 2). It is meant to summarize some conclusions about the performance of: 1) the Booster, AGS, and RHIC accelerators (and transfer lines), and 2) each polarimeter. This information will be important as input to planning for future polarized beam runs, and complements other studies of the RHIC polarimeters [1].

Topics in this note are organized as follows. Section II describes measurements made with the $200-\mathrm{MeV}$ polarimeter using both $\mathrm{C}$ and $\mathrm{CD}_{2}$ targets. The AGS polarimeter results are discussed in Sec. III. The RHIC polarimeter measurements are scrutinized in Sec. IV to evaluate their performance, and are used to draw conclusions about loss of polarization in the accelerators in Sec. V. A summary is given in Sec. VI.

\section{200-MEV POLARIMETER}

\section{A. Introduction}

Since the installation of the new optically pumped polarized ion source (OPPIS), beam currents at the end of the LINAC have increased substantially so that instantaneous rates of $200 \mu \mathrm{A}$ have become typical. This current saturated the scintillation detectors used to observe $\mathrm{p}+\mathrm{C}$ scattering at $12^{\circ}$ and $16^{\circ}$, and polarization measurements were unstable. To combat this problem, the horizontal plane detectors at $12^{\circ}$ were moved to a much larger distance. Lead shielding and collimators were added to restrict the observed charged particles to an origin at the target $[2,3]$. The size of the carbon target was reduced. At the same time, the optics of this part of the beam line were improved to make a tighter beam spot in the hope that this would reduce the contribution from scattering off thick parts of the target ladder. On a strip target such as the carbon fiber used at the LINAC, this improvement

tends to increase the rate. This raised the question of whether the calibration, made in 1982 at IUCF [4] and reported in 1989 [3], remained valid. Knowing the polarization at the end of the LINAC is crucial in evaluating whether there are significant losses in the polarization as the beam goes through the Booster and the early stages of AGS acceleration.

It was suggested that if a setup could be constructed that would observe $\mathrm{p}+\mathrm{d}$ elastic 
scattering, then it would be possible to make use of the very precise analyzing powers measured as part of the polarimeter calibration at IUCF [5, 6]. For a deuteron recoil angle of $42.6^{\circ}$ in the lab and a laboratory energy of $200 \mathrm{MeV}$, the analyzing power is known to be $A=0.507 \pm 0.002$. At the same time, the cross section at this angle is less than $1 \mathrm{mb} / \mathrm{sr}$, or about 1000 times less than that for proton inclusive scattering. So $\mathrm{p}+\mathrm{d}$ scattering cannot yield polarization values within a few minutes as the $12^{\circ}$ counters do based on $\mathrm{p}+\mathrm{C}$ scattering. One way to proceed is to use $\mathrm{p}+\mathrm{d}$ scattering in a longer run to calibrate $\mathrm{p}+\mathrm{C}$ scattering, which would then serve for routine measurements. Thus the design of the running involved interleaving $\mathrm{p}+\mathrm{C}$ and $\mathrm{p}+\mathrm{d}$ runs by swapping targets under conditions where the beam polarization was believed to be sufficiently stable to permit good time averages to be computed.

Scintillators were gathered at IUCF and mounted in a new setup. A series of $\mathrm{CD}_{2}$ targets were installed on the target ladder. During runs in July and August 2001, the initial electronics setup was tested. By August, it was possible to record one event for every pulse. A fraction of these were $\mathrm{p}+\mathrm{d}$ elastic scattering events. The $\mathrm{p}+\mathrm{d}$ events gave a reasonably clean signature in scintillator pulse height and relative timing of the deuteron and proton detector arms. To prevent signal deterioration, the LINAC beam intensity was kept below $50 \mu \mathrm{A}$ instantaneous current. Under good conditions, the polarized beam intensity can be in the $200-300 \mu \mathrm{A}$ range.

During the summer and early fall, various changes were made to the scintillators used to record $\mathrm{p}+\mathrm{d}$ scattering, mostly to remove background in the form of a high instantaneous rate of protons [2]. In the end, the deuteron detector telescope consisted of three scintillators whose thicknesses were chosen so that deuterons from $\mathrm{p}+\mathrm{d}$ scattering would stop in the second scintillator. The third then became a veto. The two proton arm detectors for each side were salvaged from the $16^{\circ} \mathrm{p}+\mathrm{C}$ setup where they had run without absorbers. They were moved to $\theta_{\text {lab }}=64.1^{\circ}$ to detect the scattered proton and a 2.54-cm thick aluminum absorber was added. Protons from $\mathrm{p}+\mathrm{d}$ scattering still traveled all the way through both detectors, but the energy deposited exceeded by a significant amount the energy from protons scattered near beam energy from carbon.

Later in the fall, the data acquisition system was upgraded to allow more events to be recorded within a single LINAC pulse, consistent with the dead times for making the analogto-digital conversion. By December, it was possible to repeat the calibration tests, and the 
$p+d$ polarimeter was used for much of the RHIC polarized beam time through the end of January.

The $\mathrm{p}+\mathrm{d}$ polarimeter system was also run at various times during the spring of 2002 to check improvements made to OPPIS. Those tests will not be covered here.

\section{B. August Calibration Run}

Over the period of a few days, $\mathrm{p}+\mathrm{C}$ scattering data at $12^{\circ}$ were collected and interspersed with periodic measurements of the beam polarization using $\mathrm{p}+\mathrm{d}$ elastic events. Data were also collected on the number of triggers in the $\mathrm{p}+\mathrm{d}$ system as well as the rates in the $12^{\circ}$ detectors with the $\mathrm{CD}_{2}$ target material in place. Neither of these proved reliable for routine polarimetry. The $\mathrm{p}+\mathrm{d}$ coincidence rate contained too many random events, and PMT gain issues discussed in Sec. II.D below rendered the $\mathrm{CD}_{2}$ rate in the forward scintillators useless.

Offline, the computer files containing $\mathrm{p}+\mathrm{d}$ events were reanalyzed [2]. Lower bounds were inserted on the acceptable pulse heights for each detector. Spectra were created to plot the time-of-arrival difference between signals in the deuteron and proton arms, and the coincident time peaks in these spectra were used to calculate the number of events for left or right scattering. A correction was made for random coincidences and backgrounds by subtracting the events recorded in a peak-width span at a time different from the $\mathrm{p}+\mathrm{d}$ peak; this correction was small.

The original calibration reported by Khiari [3] gave $A=0.620 \pm 0.004$ for $\mathrm{p}+\mathrm{C}$ inclusive scattering at $\theta_{\mathrm{lab}}=12^{\circ}$. This produced an average beam polarization of $P=0.640 \pm 0.003$. Over the same time period, $\mathrm{p}+\mathrm{d}$ runs gave a value after analysis of $P=0.688 \pm 0.015$. In order to produce such a value, the $\mathrm{p}+\mathrm{C}$ analyzing power would need to be reduced to $A=0.576 \pm 0.013$, a $7 \%$ change. This goes in the direction needed to resolve a long-standing disagreement with polarizations measured using $\mathrm{p}+\mathrm{C}$ scattering at $\theta_{\mathrm{lab}}=16^{\circ}$. For that angle, Khiari reports $A=0.511 \pm 0.004$, a value that produces a higher polarization than the $12^{\circ}$ measurements by amounts that are typically $5 \%$ larger.

Unfortunately, the newer $\mathrm{p}+\mathrm{C}$ analyzing powers now contradict a global analysis of $\mathrm{p}$ $+\mathrm{C}$ scattering reported by McNaughton [7]. His smoothed analyzing powers are $A=0.616$ at $\theta_{\mathrm{lab}}=12^{\circ}$ and $A=0.556$ at $\theta_{\mathrm{lab}}=16^{\circ}$, thus supporting the old analyzing power of Khiari at $\theta_{\text {lab }}=12^{\circ}$ and suggesting that the value at $\theta_{\text {lab }}=16^{\circ}$ is too low. 
In order to check these results for reproducibility, we decided to repeat the calibration of the LINAC polarimeter again during the spin commissioning in December 2001.

\section{December Calibration Run}

The slow ADCs and TDCs from the August calibration were replaced by FERA ADCs and readout into a CAMAC buffer. This allows several events to be recorded and stored during a single beam pulse without the overhead of CAMAC readout into a computer. The front $\Delta \mathrm{E}$ scintillators and the back veto scintillators in the deuteron arms were made smaller to reduce random rates. Incidentally, a programmable discriminator used previously to select signals from the scintillators was replaced with a fixed discriminator located in the LINAC tunnel with levels set to what was found to work best during the previous run. With the new scintillators, it was necessary to check voltage and timing settings to obtain optimum performance.

The calibration again consisted of interleaving $\mathrm{p}+\mathrm{C}$ runs with $\mathrm{p}+\mathrm{d}$ runs with the idea that the ratio of the beam polarizations measured with these two systems was a check of the $\mathrm{p}+\mathrm{C}$ calibration. Again, scaler readout with the $\mathrm{CD}_{2}$ target was tested using both $\mathrm{p}$ $+\mathrm{C}$ rates at $12^{\circ}$ and $\mathrm{p}+\mathrm{d}$ coincident rates (see Sec. II.B) to see whether either of these could also serve as a measure of the beam polarization. The situation with these rates had not improved.

In order that this operation be practical during the AGS polarized beam development, periodic switching of the beam was implemented from the Booster to the straight-through line at the LINAC where the polarimeter is located. The rate for the $\mathrm{p}+\mathrm{d}$ system was still too large, and therefore one of the quadrupoles was defocussed, also in beam sharing mode, so that the calibration was made at a smaller current. This procedure may increase detector rates if the tails of the beam reach the target frame.

Despite improvements to the data acquisition, the overall $\mathrm{p}+\mathrm{d}$ rate turned out again to be low. In part, this was due to the fact that with beam sharing, only 1 pulse every 5 seconds was available in the polarimeter line. Second, for reasons discussed in more detail in Sec. II.D, rate problems with the $\mathrm{p}+\mathrm{d}$ scintillator systems forced operation at the edge of the beam spot where currents were effectively less than about $20 \mu \mathrm{A}$, again cutting rate. Thus this calibration was spread out over four days of running. 
During the calibration, beam polarizations recorded by $\mathrm{p}+\mathrm{C}$ scattering varied between 0.67 and 0.71 . These variations were treated as real, and could be associated with changes in the operation of the ion source between pulsed and DC mode in the ionizer. The changes from one mode to another served to keep the ionizer cleaner, and this helped to maintain a higher polarization. Beam polarization ratios between $\mathrm{p}+\mathrm{C}$ and $\mathrm{p}+\mathrm{d}$ scattering were calculated only for runs that came together during a single mode. These ratios were averaged over the whole calibration.

The electronics was set so that only the higher pulse height signals associated with $\mathrm{p}+$ $\mathrm{d}$ scattering were allowed to generate the logic signals going into the coincidence between the deuteron and proton arms. Additional, even higher cuts were placed on the pulse height signals for analysis. Upper cuts were avoided since there was evidence for pileup of real events in the pulse height spectra. The time peak for $\mathrm{p}+\mathrm{d}$ scattering remained narrow. Summing was made in the time spectrum, gated on correct scintillator pulse height. A small background of randoms, no more than a few percent, was subtracted from the time peak as needed.

The ratio of the beam polarization measured with $\mathrm{p}+\mathrm{d}$ scattering to that measured with $\mathrm{p}+\mathrm{C}$ scattering was found to be

$$
P_{\mathrm{pd}} / P_{\mathrm{pC}}=0.987 \pm 0.017
$$

a value consistent with one. Detailed polarimeter measurements for each stable ion source cycle are listed in Table I. Because systematic errors are likely to increase this statistical error, there is no recommendation from this calibration that the analyzing power used in the $\mathrm{p}+\mathrm{C}$ analysis be changed. But the previous ratio of $P_{\mathrm{pd}} / P_{\mathrm{pC}}=1.075 \pm 0.024$ from the August calibration was not reproduced. For both runs the polarization measured with $\mathrm{p}+$ C scattering was similar.

\section{Systematic Errors}

The main difficulty with both the $\mathrm{p}+\mathrm{C}$ and $\mathrm{p}+\mathrm{d}$ systems was the sensitivity of the photomultiplier tubes to rate.

In the $p+d$ case, excessive rate would cause the gain to drop, and the signals of interest would fall below the discriminator cutoff. Under different running conditions, gains were 
TABLE I: Partial Results from December Calibration

\begin{tabular}{|c|c|c|c|}
\hline Cycle & $P_{p C}$ & $P_{p d}$ & $P_{p d} / P_{p C}$ \\
\hline 1 & $0.673 \pm 0.004$ & $0.663 \pm 0.023$ & $0.986 \pm 0.035$ \\
2 & $0.703 \pm 0.006$ & $0.693 \pm 0.044$ & $0.986 \pm 0.063$ \\
3 & $0.673 \pm 0.005$ & $0.688 \pm 0.024$ & $1.023 \pm 0.036$ \\
4 & $0.722 \pm 0.007$ & $0.776 \pm 0.044$ & $1.074 \pm 0.062$ \\
5 & $0.722 \pm 0.007$ & $0.802 \pm 0.048$ & $1.112 \pm 0.067$ \\
6 & $0.710 \pm 0.005$ & $0.656 \pm 0.020$ & $0.924 \pm 0.029$ \\
\hline average & & & $0.987 \pm 0.017$ \\
\hline
\end{tabular}

observed to change by factors of up to three. In all cases, measurements were made at rates that caused the PMT gain to saturate, a phenomenon that could easily be observed by tracking the falling signal pulse height with time in the LINAC pulse. During the August run, stable but still saturated conditions were found with currents of $50 \mu \mathrm{A}$. In December with the beam current still $50 \mu \mathrm{A}$, the $\mathrm{CD}_{2}$ target was placed at the edge of the beam so that the effective current was in the range of $10-20 \mu \mathrm{A}$. Only at this level was it possible to keep the $\mathrm{p}+\mathrm{d}$ signals above threshold with enough efficiency that the rates for left and right scattering were comparable. Some short runs were made in which the efficiency of the right-hand system was lower than the left-hand system by perhaps a factor of two. In these cases, there is some indication (weak statistics) that the polarization is systematically larger. Figure 1 shows that the scatter plot of $\mathrm{p}+\mathrm{d}$ polarization tends to rise with positive geometrical asymmetry $\left(\epsilon_{\text {geom }}\right.$ - see Sec. IV; unpolarized rates to beam left exceed those to beam right). This effect has this sign because the $\mathrm{p}+\mathrm{d}$ analyzing power is negative (we use a positive $A$ for the deuteron) while proton inclusive analyzing powers are positive.

For the $\mathrm{p}+\mathrm{C}$ system, there is also a reduction in photomultiplier gain with increasing rate. Here the effects are not as severe. Since the positive analyzing power is associated with the primary proton rate into these scintillators, the effect of reduced gain shows up as a smaller polarization value. A number of tests were made during the course of the December run comparing $\mathrm{p}+\mathrm{C}$ measurements at beam currents between 50 and $200 \mu \mathrm{A}$. Figure 2 shows two such scans in which the beam current was controlled by defocussing LINAC quad 14 (solid points) or closing slits near OPPIS (open points). The horizontal axis is scintillator 


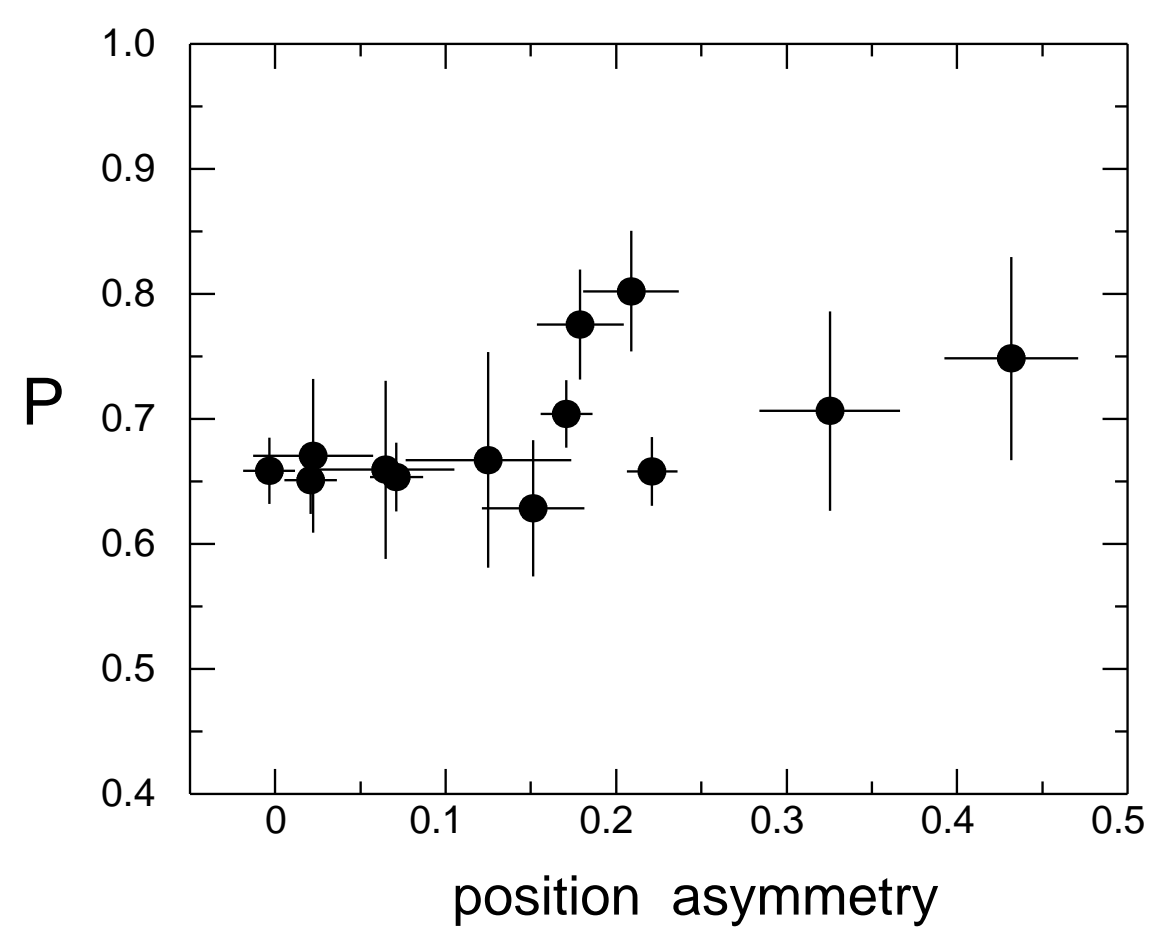

FIG. 1: Correlation between $\mathrm{p}+\mathrm{d}$ polarizations measured during the December run and the geometrical or position asymmetry, "( $\mathrm{L}-\mathrm{R}) /(\mathrm{L}+\mathrm{R})$ ", for the rates recorded by the left and right scattering systems. Note that these are very large position asymmetries, as discussed in the text.

rate in arbitrary units. For both scans, asymmetries are divided by the asymmetry at the lowest rate (37) to show the percentage reduction. A linear fit is included as a guide to the eye. (The rate values correspond roughly to the LINAC beam current in $\mu \mathrm{A}$.) Additional checks gave qualitatively similar results. It is important to keep these changes in mind when interpreting the results of $\mathrm{p}+\mathrm{C}$ polarization measurements.

The experience with rate limitations suggests that there are systematic effects whose contribution to the calibration error exceeds the statistical error quoted above. An estimate of 0.02 or even 0.03 would appear to be appropriate, so $P_{\mathrm{pd}} / P_{\mathrm{pC}} \cong 0.987 \pm 0.03$.

It would be desirable to have polarization measurements that are less sensitive to beam currents for currents typically used at the LINAC. For this, it will be necessary to further alter both the $\mathrm{p}+\mathrm{C}$ and $\mathrm{p}+\mathrm{d}$ systems to be more rate tolerant. 


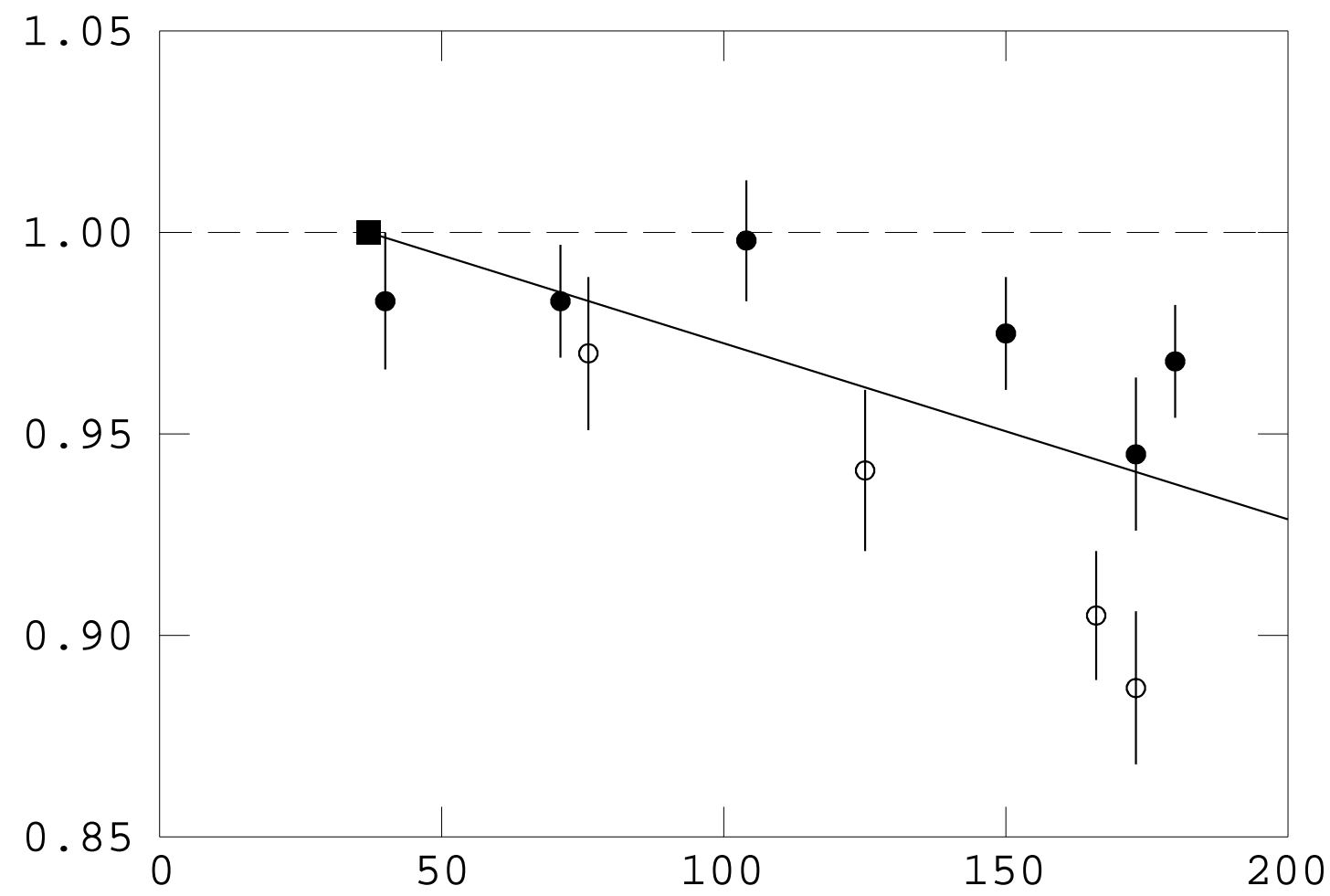

FIG. 2: Ratio of the $\mathrm{p}+\mathrm{C}$ asymmetry, as a function of the $\mathrm{p}+\mathrm{C}$ rate, to the asymmetry measured at the lowest rate. The beam currents were adjusted by defocussing LINAC quad 14 (solid circles) or closing slits (open circles). The dashed line would correspond to no change in asymmetry with rate, and the solid line is a linear fit to all the data. The rates on the horizontal axis correspond roughly to the LINAC beam current in $\mu \mathrm{A}$.

\section{AGS POLARIMETER}

Upgrades were made to the AGS (E880) polarimeter for this run period. Six additional plastic scintillation counters were added to detect the forward scattered protons for low beam momenta. The goal was to perform an absolute calibration of the polarimeter at $G \gamma=7.5$ $\left(p_{l a b}=3.8 \mathrm{GeV} / \mathrm{c}\right)$. The measurements and results are described in a separate note; see Ref. [8]. No variations outside statistics were observed for asymmetries in $G \gamma=7.5$ runs that were close in time.

Another upgrade was the addition of some new VME scalers, which were connected to the C-AD data acquisition system. These scalers recorded a few of the quantities generated 


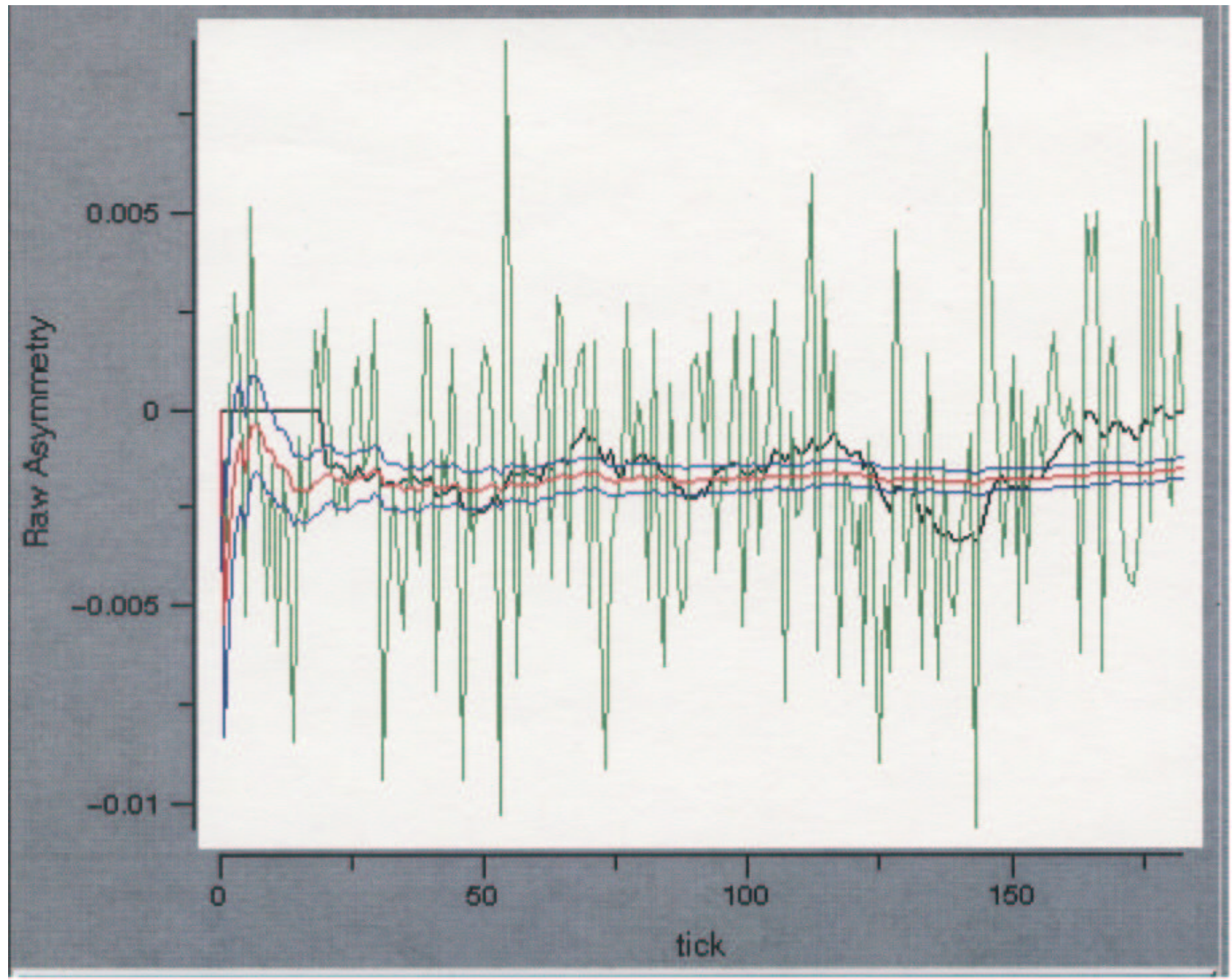

FIG. 3: Plot of the time dependence of the E880 polarimeter asymmetry within a run. The lines correspond to the running average and statistical errors, and the 20 spill average. This run lasted about 15 minutes.

by the polarimeter electronics; many more scalers were used in the data acquisition system dedicated to the polarimeter. Software to read the VME scalers, compute asymmetries, and display them during a run was written by $\mathrm{H}$. Huang and A. Marušić. These displays all showed smooth time variation, see Fig. 3. It had been suggested that some of the changes in asymmetries measured by the AGS polarimeter were caused by intermittent anomalous behavior of the hardware, but there was no evidence for this in the displays. For instance, no discontinuities in the running average asymmetries were observed. (Square root asymmetries were used for all AGS polarimeter results described in this section; see also Sec. IV.) 
The AGS polarimeter was operated under similar conditions (photomultiplier tube high voltages, electronic logic, wedge positions, and carbon fiber target) as in previous years. Rate effects were low with the thin carbon fiber targets, as verified by observing pulse heights in the polarimeter counters on an oscilloscope during a spill. Target position scans were performed to insure that the beam center was far enough from the fishline target so that there were only events from the carbon fibers recorded in the data. The maximum asymmetries recorded at $G \gamma=7.5,12.5,30.5$, and 46.5 are shown in Fig. 4 as a function of time. Using the approximate analyzing powers

$$
\begin{aligned}
A_{N} & \sim 0.0456 \pm 0.0029 \text { for } G \gamma=7.5 \\
A_{N} & \sim 0.025 \pm 0.003 \quad \text { for } \quad G \gamma=12.5 \\
A_{N} & \sim 0.0093 \pm 0.0015 \text { for } G \gamma=30.5 \\
A_{N} & \sim 0.0058 \pm 0.0012 \text { for } \quad G \gamma=46.5
\end{aligned}
$$

it can be seen that beam polarizations were typically $\sim 70 \%$ at $G \gamma=7.5$, but only $~$ $25 \%$ at $G \gamma=46.5$, or the extraction energy. The asymmetry values at $G \gamma=12.5$ varied significantly more than at $G \gamma=7.5$, which suggests the polarization loss between the two energies varied from time to time. One should note that some of the measurements at $G \gamma=12.5$ and 30.5 were made without tuning the AGS parameters (betatron tunes, AC dipole settings, ...) for the measuring flattop. Thus, they may not reflect the true polarization level for the extraction magnet cycle. The slower AGS ramp rate, imposed by the use of the Westinghouse instead of the Siemens motor generator (MG) set, was likely responsible for the larger polarization losses in 2001/2002 (RHIC run 2) than seen previously. Also, the E880 polarimeter calibrations above at $G \gamma>7.5$ have a much larger uncertainty than the $5 \%$ desired for RHIC.

Finally, searches were made for possible systematic errors. Correlations of the physics asymmetry ( $\epsilon_{\text {phys }}=\epsilon_{A G S}$, see Eq. 6 below) with the luminosity $\left(\epsilon_{\text {lum }}\right.$, Eq. 8$)$ or the geometrical ( $\epsilon_{\text {geom }}$, Eq. 9) asymmetries are shown in Figs. 5 and 6. Only six sets of five or more runs with constant operating conditions were found among the $\sim 1400$ AGS polarimeter measurements during RHIC run 2. One set was from 16 December 2001, while the others occurred on 13 - 23 January 2002, with typically a day or two between these sets. Runs taken during RHIC filling had to be excluded as the number of + and - spills were usually 

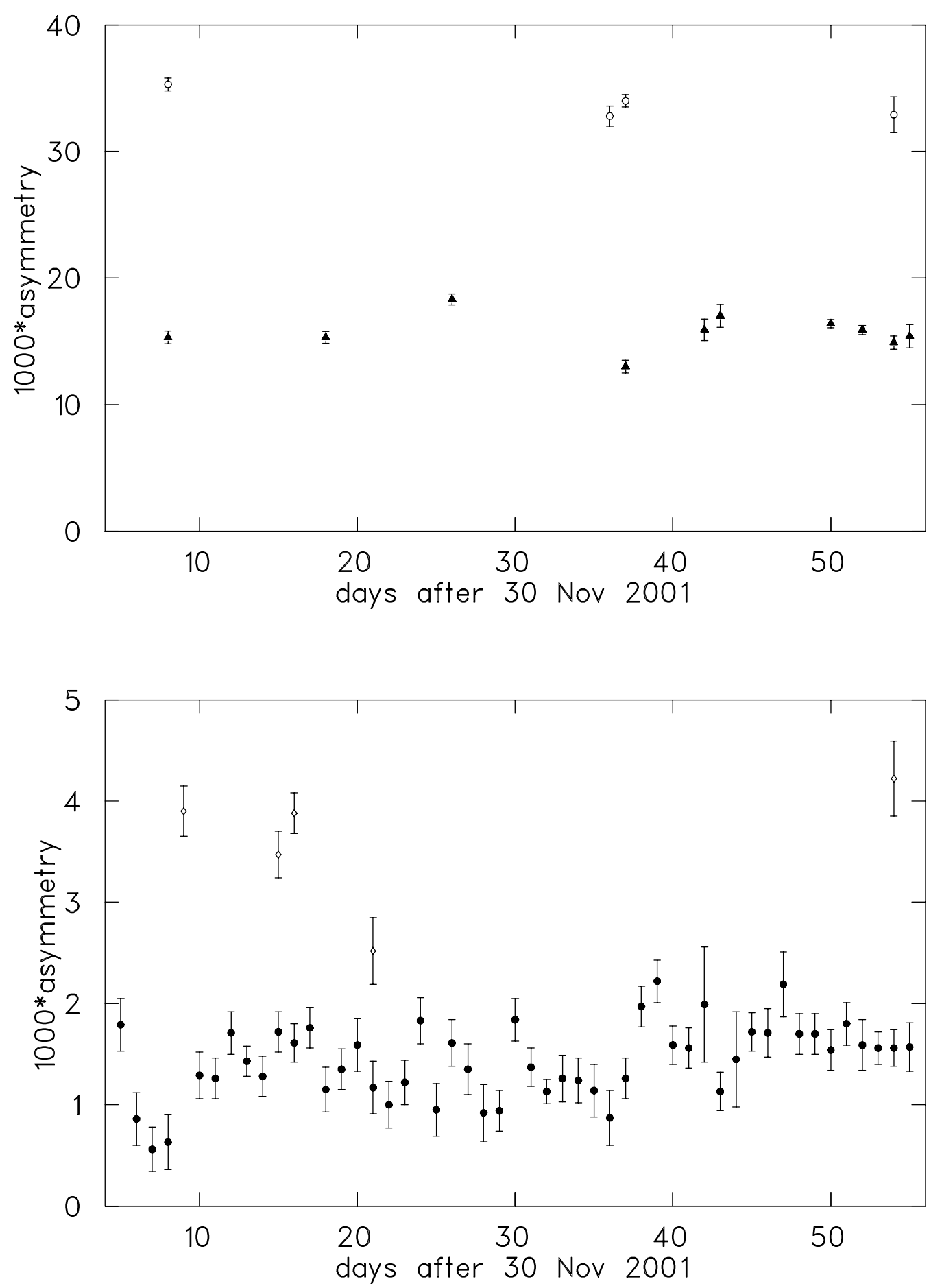

FIG. 4: Maximum asymmetry measured with the AGS polarimeter as a function of day. The upper plot includes $G \gamma=7.5$ (open circles) and 12.5 (solid triangles), and the lower plot has $G \gamma=30.5$ (open diamonds) and 46.5 (solid circles). Some additional points at $G \gamma=12.5$ are not shown since they were collected with the AC dipole off. 

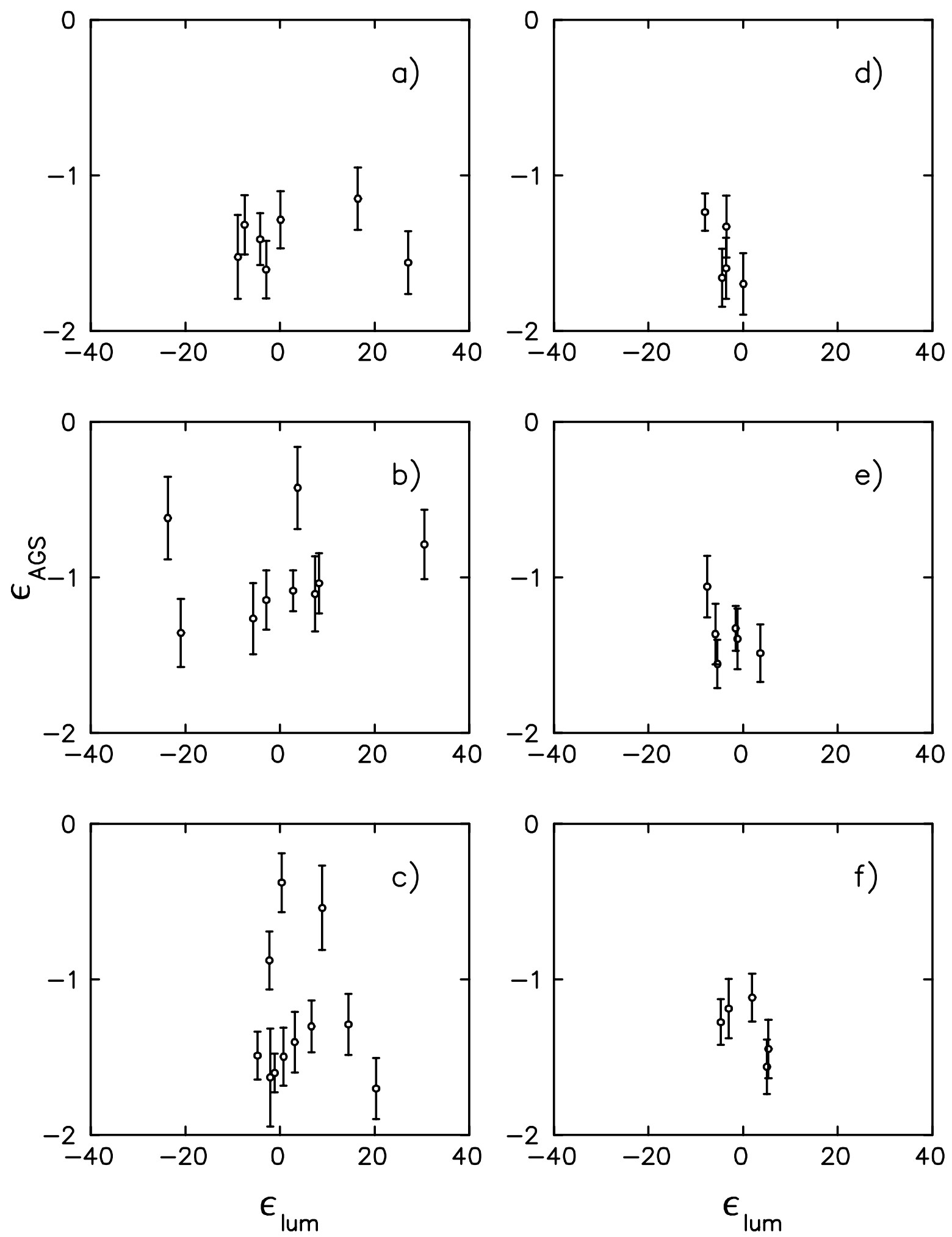

FIG. 5: Correlation of the AGS polarimeter physics asymmetry with the luminosity asymmetry for six sets of runs taken under approximately constant conditions. All asymmetries have been multiplied by $10^{3}$. 

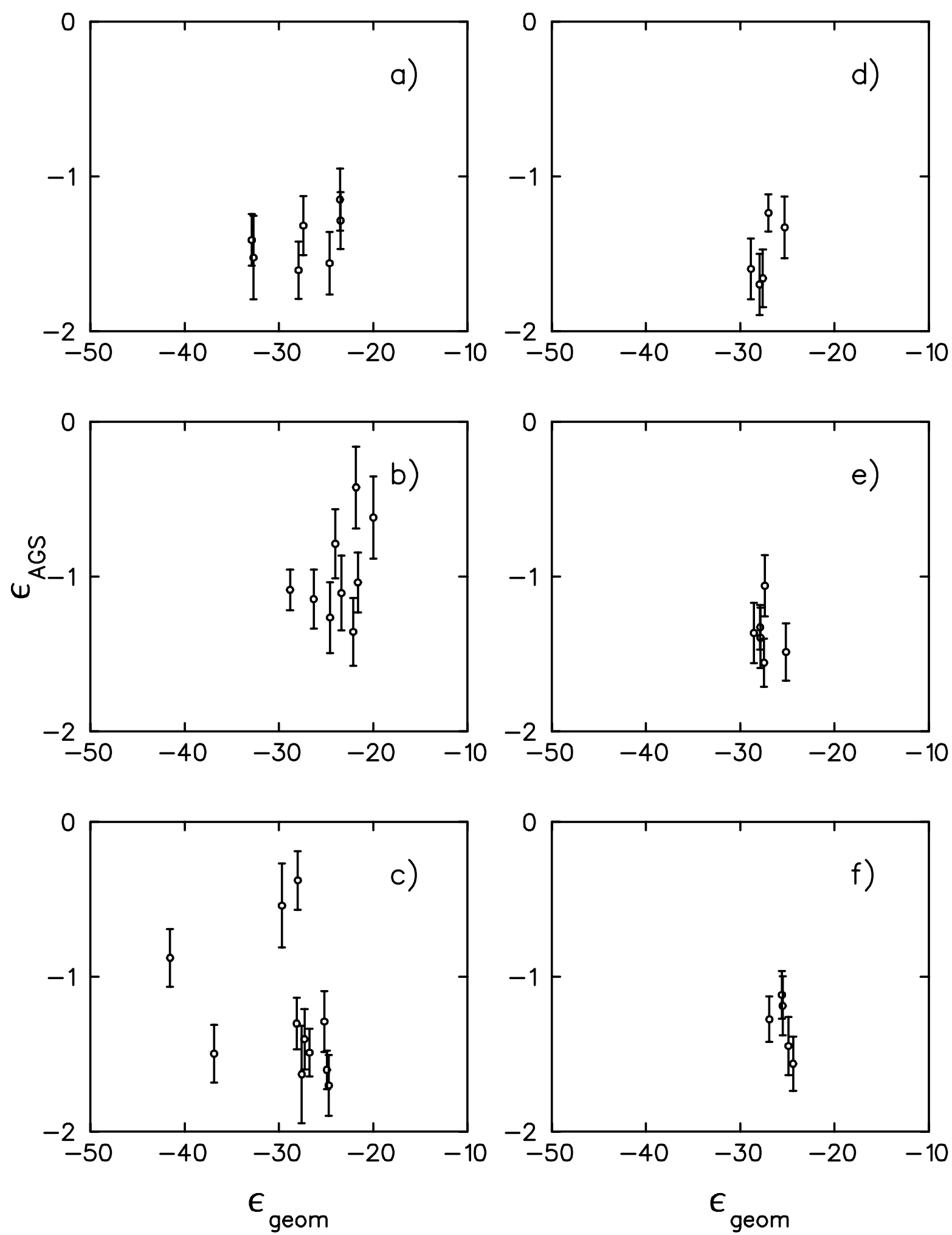

FIG. 6: Correlation of the AGS polarimeter physics asymmetry with the geometrical asymmetry for six sets of runs taken under approximately constant conditions. All asymmetries have been multiplied by $10^{3}$. 
very different, suggesting that the spin direction stayed unchanged for a long period.

No statistically significant correlation of $\epsilon_{A G S}$ with $\epsilon_{\text {lum }}$ is apparent in Fig. 5 for any of the data sets, or for the combined data (not shown). There is also no correlation of $\epsilon_{A G S}$ with $\epsilon_{\text {geom }}$ observed in any of the sets in Fig. 6, but one is seen for the combined sets. This latter observation is not considered significant since there were different AGS operating conditions for each set, and drifts in $\epsilon_{\text {geom }}$ with time occurred. It is concluded that no evidence of correlations exists. However, while the existence of even a weak correlation would signal the presence of a systematic error, the absence of an observable correlation does not demonstrate the lack of systematic effects.

\section{RHIC POLARIMETER PERFORMANCE}

A number of tests were made searching for systematic effects in the RHIC polarimeter data. These began with the tabulated data (O. Jinnouchi and K. Kurita) from RHIC run 2, removing results with known hardware problems or special operating conditions. The tests are described in the sections below.

\section{A. Luminosity Asymmetry Differences}

A search was made for systematic effects in the RHIC polarimeter data using luminosity asymmetries. For (approximately) symmetric sets of detectors on opposite sides of the beam ( $\mathrm{L}=$ left, $\mathrm{R}=$ right), the number of counts recorded in some time for idealized detectors will be

$$
\begin{aligned}
& N_{L}^{\uparrow}=N_{0} B^{\uparrow} d \Omega_{L}\left(1+P^{\uparrow} A_{L}\right) \\
& N_{L}^{\downarrow}=N_{0} B^{\downarrow} d \Omega_{L}\left(1-P^{\downarrow} A_{L}\right) \\
& N_{R}^{\uparrow}=N_{0} B^{\uparrow} d \Omega_{R}\left(1-P^{\uparrow} A_{R}\right) \\
& N_{R}^{\downarrow}=N_{0} B^{\downarrow} d \Omega_{R}\left(1+P^{\downarrow} A_{R}\right),
\end{aligned}
$$

where the integrated beam intensities are $B^{\uparrow}$ and $B^{\downarrow}$, and beam polarizations are $P^{\uparrow}$ and $P^{\downarrow}$ for spin directions up $(\uparrow)$ and down $(\downarrow)$, respectively. The analyzing powers for the $L$ and $R$ detectors are $A_{L}$ and $A_{R}$, and the products of solid angle and efficiency are $d \Omega_{L}$ and $d \Omega_{R}$. 
A normalization factor, $N_{0}$, is dependent on running time, cross section, target thickness, etc.

The "physics asymmetry" can be determined from the square root formula

$$
\begin{aligned}
\epsilon_{\text {phys }} & =\frac{\sqrt{N_{L}^{\uparrow} N_{R}^{\downarrow}}-\sqrt{N_{L}^{\downarrow} N_{R}^{\uparrow}}}{\sqrt{N_{L}^{\uparrow} N_{R}^{\downarrow}}+\sqrt{N_{L}^{\downarrow} N_{R}^{\uparrow}}} \\
& =\frac{1}{2}\left(P^{\uparrow}+P^{\downarrow}\right) \times \frac{1}{2}\left(A_{L}+A_{R}\right)+\text { h.o.t. } \\
& =P A+\text { h.o.t. }
\end{aligned}
$$

where the higher order terms (h.o.t.) are fourth order in the five small quantities

$$
\begin{aligned}
\epsilon_{A} & =\frac{A_{L}-A_{R}}{A_{L}+A_{R}} \\
\epsilon_{B} & =\frac{B^{\uparrow}-B^{\downarrow}}{B^{\uparrow}+B^{\downarrow}} \\
\epsilon_{d \Omega} & =\frac{d \Omega_{L}-d \Omega_{R}}{d \Omega_{L}+d \Omega_{R}} \\
\epsilon_{P} & =\frac{P^{\uparrow}-P^{\downarrow}}{P^{\uparrow}+P^{\downarrow}} \\
P A & =\frac{1}{2}\left(P^{\uparrow}+P^{\downarrow}\right) \times \frac{1}{2}\left(A_{L}+A_{R}\right) .
\end{aligned}
$$

For relatively low energies, $P A$ can be quite large, and bigger than $\epsilon_{A}, \epsilon_{B}, \epsilon_{d \Omega}$, and $\epsilon_{P}$. However, at RHIC energies, $P A$ is generally much smaller; for the CNI polarimeters, $P A$ may actually be second order compared to the other four quantities in Eq. 7 .

Another type of square root asymmetry, the "luminosity asymmetry" $\epsilon_{\text {lum }}$, can be formed which is sensitive to $\epsilon_{B}$

$$
\begin{aligned}
\epsilon_{\text {lum }} & =\frac{\sqrt{N_{L}^{\uparrow} N_{R}^{\uparrow}}-\sqrt{N_{L}^{\downarrow} N_{R}^{\downarrow}}}{\sqrt{N_{L}^{\uparrow} N_{R}^{\uparrow}}+\sqrt{N_{L}^{\downarrow} N_{R}^{\downarrow}}} \\
& =\epsilon_{B}+P A \epsilon_{A}+\text { h.o.t., }
\end{aligned}
$$

where the higher order terms are now third order in small quantities.

A third type of square root asymmetry, the "geometrical asymmetry" $\epsilon_{\text {geom }}$, is sensitive to $\epsilon_{d \Omega}$

$$
\epsilon_{\text {geom }}=\frac{\sqrt{N_{L}^{\uparrow} N_{L}^{\downarrow}}-\sqrt{N_{R}^{\uparrow} N_{R}^{\downarrow}}}{\sqrt{N_{L}^{\uparrow} N_{L}^{\downarrow}}+\sqrt{N_{R}^{\uparrow} N_{R}^{\downarrow}}}
$$




$$
=\epsilon_{d \Omega}+P A \epsilon_{p}+\text { h.o.t. }
$$

with the higher order terms also third order in small quantities.

There are three pairs of detectors in each RHIC polarimeter, with each detector of the pair on opposite sides of the beam, labeled 1-4, 2-5 (X90), and 3-6. Detectors 2 and 5 are in the horizontal plane, while the others are $\pm 45^{\circ}$ out of this plane. These six detectors view the same polarimeter target and beam. Therefore, the luminosity asymmetries of the three pairs should agree within statistical uncertainties. Significant deviations would suggest systematic errors in $\epsilon_{\text {lum }}$, and perhaps also in the physics asymmetry.

As an example, consider a hardware problem in the $L$ detector only, such that

$$
\begin{aligned}
& N_{L}^{\uparrow} \rightarrow N_{L}^{\uparrow}(1+\delta) \\
& N_{L}^{\downarrow} \rightarrow N_{L}^{\downarrow}(1-\delta),
\end{aligned}
$$

perhaps due to a rate dependence in the $L$ detector response and differences in intensity in the $\uparrow$ and $\downarrow$ beams. Then

$$
\begin{aligned}
\epsilon_{\text {lum }} & \rightarrow \epsilon_{\text {lum }}+\delta / 2 \\
\epsilon_{\text {phys }} & \rightarrow \epsilon_{\text {phys }}+\delta / 2,
\end{aligned}
$$

or the magnitudes of the systematic effects may be comparable in $\epsilon_{\text {lum }}$ and $\epsilon_{\text {phys }}$.

A search was made for such differences in the luminosity asymmetry from the three pairs of detectors. The polarimeter runs were separated into a set for the Blue and a set for the Yellow ring. Any differences of more than four standard deviations (for the larger statistical error $\sigma$ of the pair) were recorded. A total of 360 and 320 runs were considered for the Blue and Yellow rings, respectively, and the number of runs with such large differences was 78 and 87. The number of such runs expected from statistical fluctuations of $4 \sigma$ was 1 - 2 . Clearly, systematic effects are present in the data.

The observed differences in $\epsilon_{\text {lum }}$ were further studied as a function of time using the data in the RHIC polarimeter data spreadsheet version 1.2. Figure 7 gives the magnitude of the observed luminosity asymmetry differences as a function of run number with Blue and Yellow ring results combined. These are compared to four times the statistical error for the 2-5 asymmetry, $4 \sigma_{X 90}$. The differences are subdivided into sets where the 1-4 detector pair 


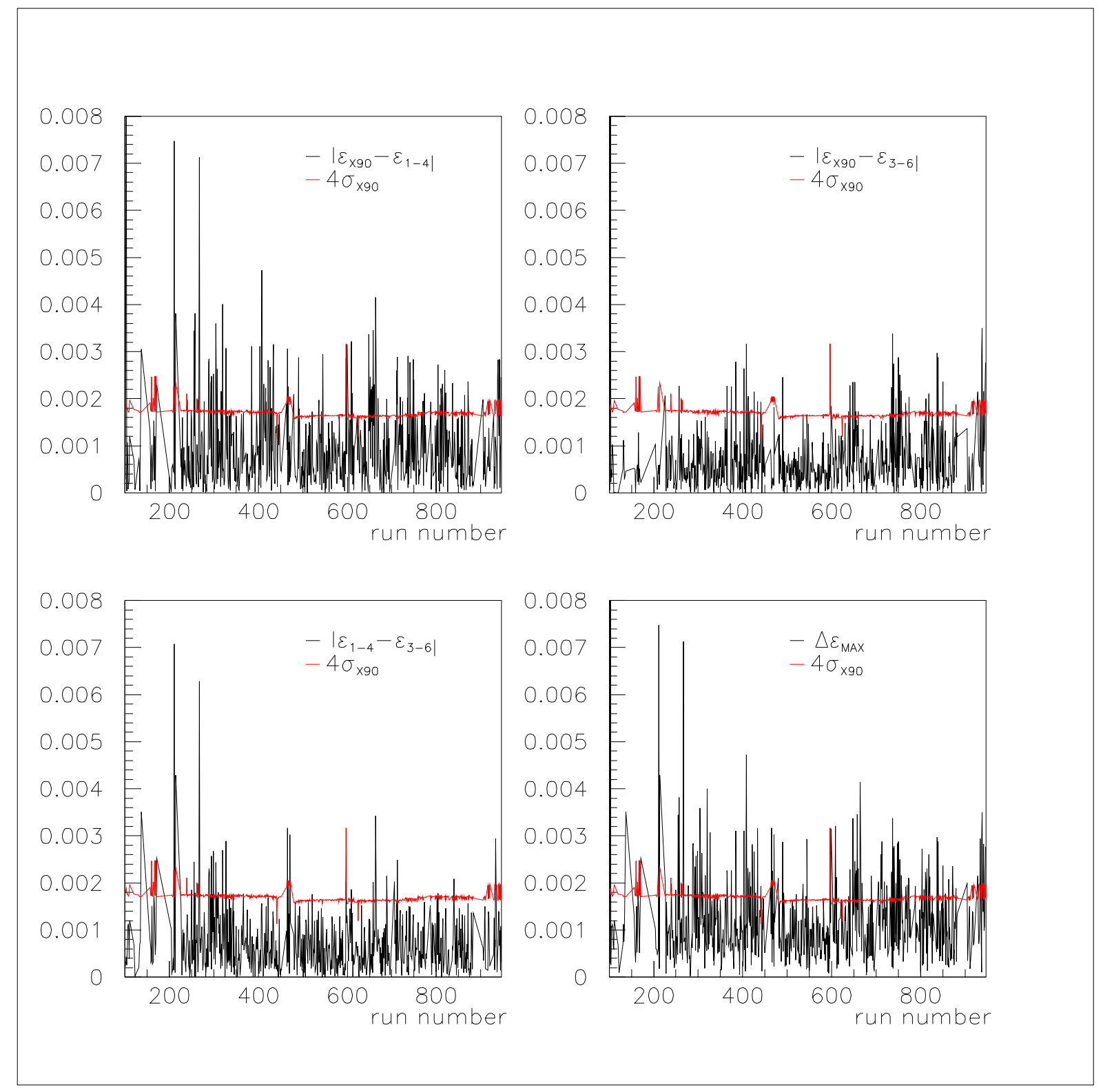

FIG. 7: These plots show the differences between the luminosity asymmetries as a function of RHIC polarimeter run number. The lower right plot shows the maximum of the three differences that can be formed. The differences are compared to $4 \sigma_{X 90}$, where $\sigma_{X 90}$ is the statistical error of $\epsilon_{X 90}=\epsilon_{2-5}$ 


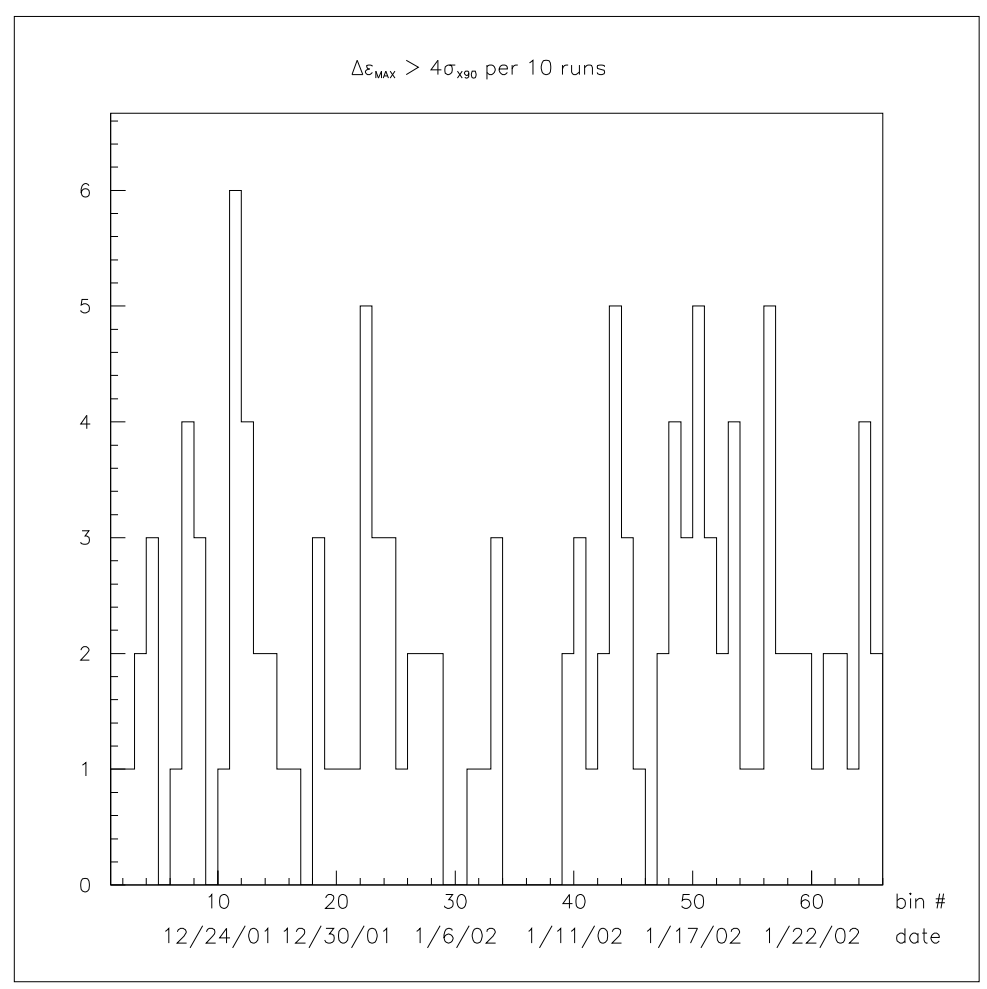

FIG. 8: This plot shows fluctuations in the number of large $\Delta \epsilon_{M A X}$ values over time. Each bin represents the number of times $\left|\Delta \epsilon_{M A X}\right|$ is greater than $4 \sigma_{X 90}$ per 10 runs.

asymmetry is compared to that for the 2-5 pair, etc., as well as the maximum difference. Note that more than one $4 \sigma_{X 90}$ difference, may occur per run. Finally, the number of cases where the maximum asymmetry difference, $\Delta \epsilon_{M A X}$ exceeds $4 \sigma_{X 90}$ per 10 runs is histogrammed in Fig. 8. These results suggest that problems occurred throughout the RHIC run period, with some times a bit worse than others.

Figure 9 shows similar results to Fig. 8, but separately for each beam and asymmetry difference. In this case, version 1.1 of the RHIC polarimeter spreadsheet and 20 runs per bin were used. It can be seen that there were no long sets of runs without any $4 \sigma$ differences, the maximum being 94 runs in the Blue ring for the 2-5 vs. 3-6 difference. Also, the differences occurred much more frequently between the 1-4 and 2-5 detector pairs than for the other two differences, for both the Blue and Yellow rings.

The runs with large luminosity asymmetry differences were studied in more detail. Of the 80 such runs after run \#600 on 10 January 2002, 26 were at injection and 25 were the first 
Blue Ring
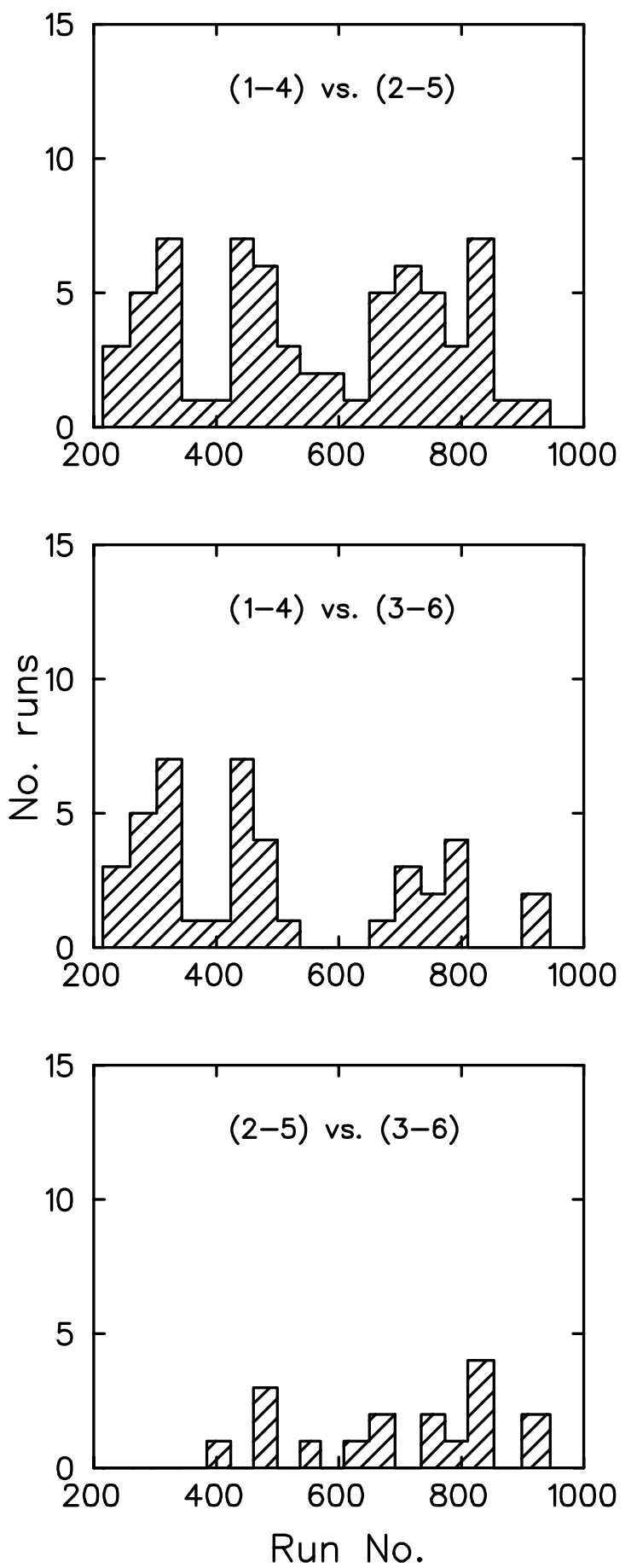

Yellow Ring
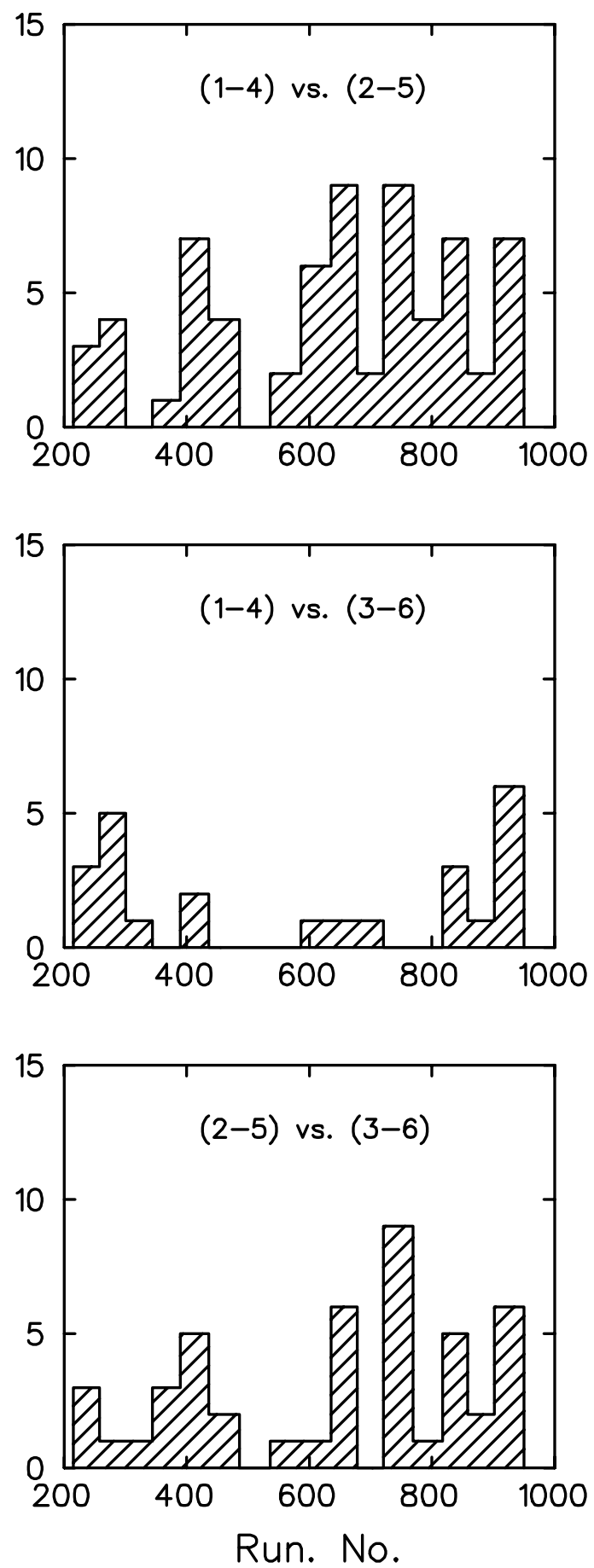

FIG. 9: The number of runs with $4 \sigma$ differences in luminosity asymmetries from various pairs of detectors in the RHIC polarimeters as a function of run number. Each bin corresponds to 20 runs tested. Separate histograms are shown for the Blue and Yellow rings, and for the comparison of $\epsilon_{\text {lum }}$ for 1-4 and 2-5, etc. pairs. Based on statistical fluctuations, an average of $\sim 0.09$ per 20 run bin is expected. 
measurement on flattop. About 1/3 (25) were in one of five RHIC fills, where the problems occurred predominantly in one ring. Finally, the average data collection time for runs with big luminosity asymmetry differences was slightly less than for runs without such differences (43 vs. 48 sec.). These observations are all suggestive of higher average rates in runs with luminosity asymmetry problems.

There were many runs where big discrepancies were observed in both 1-4 vs. 2-5 and 1-4 vs. 3-6 for the Blue ring, or for both 1-4 vs. 2-5 and 2-5 vs. 3-6 for the Yellow ring. This evidence suggests that the systematic effects are generally worse in the 1-4 detector pair for the Blue ring and the 2-5 detector pair for the Yellow ring. No characteristic or behavior of the hardware has been found that correlates with the previous observation. Although there are smaller numbers of other differences in $\epsilon_{l u m}$, these are still more than expected from statistical fluctuations. For example, the observed fractions of these differences, $\operatorname{Prob}\left(\left|\operatorname{Diff}_{i}\right| / \sigma_{i}>4\right)$, range from $17 / 360=0.047$ to $67 / 320=0.209$. Assuming the statistical uncertainties for all three luminosity asymmetries are the same (the 2-5 uncertainties are generally slightly larger than the other two), then the expected fraction is

$$
\begin{aligned}
\operatorname{Prob}\left(\left|\operatorname{Diff} f_{i}\right| / \sigma_{i}>4\right) & \simeq \operatorname{Prob}\left(\left|\operatorname{Diff} f_{i}\right| / \sigma_{\text {Diff }, i}>2 \sqrt{2}\right) \\
& \simeq 0.0046
\end{aligned}
$$

Thus, the measurements give such differences at rates more than 10 times larger than expected statistically. Another way of expressing this is as follows. Each section of Fig. 9 would be expected to have (0.0046)(360 runs) $=1.7$ large differences for the Blue ring, and $(0.0046)(320$ runs $)=1.5$ for the Yellow ring, whereas 17 - 67 are actually observed.

As noted earlier, the magnitude of systematic effects may be comparable in $\epsilon_{p h y s}$ and $\epsilon_{\text {lum }}$. Assuming they are of similar size, then the systematic error on $\epsilon_{\text {phys }}, \delta \epsilon_{\text {phys,sys }}$, can be estimated. In order for the observed number of large $\epsilon_{\text {lum }}$ differences to be consistent with statistics, the total uncertainty would need to increase about a factor of 2.2 :

$$
\operatorname{Prob}\left(\left|\operatorname{Diff}_{i}\right| / \sigma_{\text {Diff }, i}^{\prime}>2 \sqrt{2} / 2.2\right) \sim 0.20
$$

or

$$
\sigma_{D i f f, i}^{\prime} \simeq 2.2 \sigma_{D i f f, i}
$$

The typical physics asymmetries during the past RHIC polarized $p p$ run were $\epsilon_{p h y s} \sim(2-$ $3) \times 10^{-3}$, and statistical uncertainties were $\delta \epsilon_{p h y s} \sim \pm 0.38 \times 10^{-3}$. The combined statistical 
and systematic error would be approximately 2.2 times bigger, so

$$
\begin{aligned}
\delta \epsilon_{\text {phys }, \text { sys }} & \sim \sqrt{(2.2)^{2}-1} \delta \epsilon_{\text {phys }}=1.96 \delta \epsilon_{\text {phys }} \\
& \sim \pm 0.74 \times 10^{-3} .
\end{aligned}
$$

Thus, the systematic error is roughly $30 \%$ of $\epsilon_{\text {phys }}$, which is substantially larger than the goal of $<5 \%$. (With an analyzing power $A \sim 0.013$, this corresponds to $\delta P \sim \pm 0.057$, or larger than $5 \%$ of a 0.70 beam polarization.) Since the origin(s) of these systematic effects is not presently understood, it is uncertain how this systematic error would change when $\epsilon_{\text {phys }}$ increases. In conclusion, systematic effects in $\epsilon_{l u m}$ and perhaps comparable effects in $\epsilon_{\text {phys }}$ have been observed that may be much larger than the desired $5 \%$ of $\epsilon_{\text {phys }}$. It is important that further detailed studies be undertaken to attempt to identify the source of these systematic effects, using the hints provided by this analysis as well as other information.

\section{B. Other Searches for Systematic Effects}

There was other evidence of systematic problems in the RHIC polarimeter data. Since the beam polarization direction was vertical in RHIC run 2, the up-down physics asymmetry was expected to vanish. There were no RHIC polarimeter detectors in the purely up or down directions, so the detectors at $\pm 45^{\circ}$ were used to perform this measurement. Thus, "up" was taken to be the sum of counts from detectors 1 and 6 , while "down" was the sum of counts

from detectors 3 and 4, and the physics asymmetry (Eq. 6) was labeled $\epsilon_{Y 45}$. A plot of $\epsilon_{Y 45}$ divided by its statistical error is shown in Fig. 10 for the Yellow ring. Clearly, some of the measurements are nonzero. It can also be seen that the mean value of $\epsilon_{Y 45}$ fluctuates with time, changing from generally positive to usually negative about 18 January 2002. Taken at face value, the observed values of $\epsilon_{Y 45}$ and $\epsilon_{X 45}$ would suggest the spin direction was about $15-20^{\circ}$ from vertical on 20 January 2002.

Another combination of counts from the $45^{\circ}$ detectors is expected to vanish. In this case, "left" was the sum of counts from detectors 1 (up, left) and 4 (down, right), and "right" was the sum from detectors 3 (down, left) and 6 (up, right). The corresponding physics asymmetry (Eq. 6) was designated $\epsilon_{c r 45}$, and it is expected to be zero since "left" and "right" each include contributions from all four directions. Histograms of the ratio of $\epsilon_{c r 45}$ and $\epsilon_{Y 45}$ to their corresponding statistical uncertainties for most polarimeter runs (excluding 


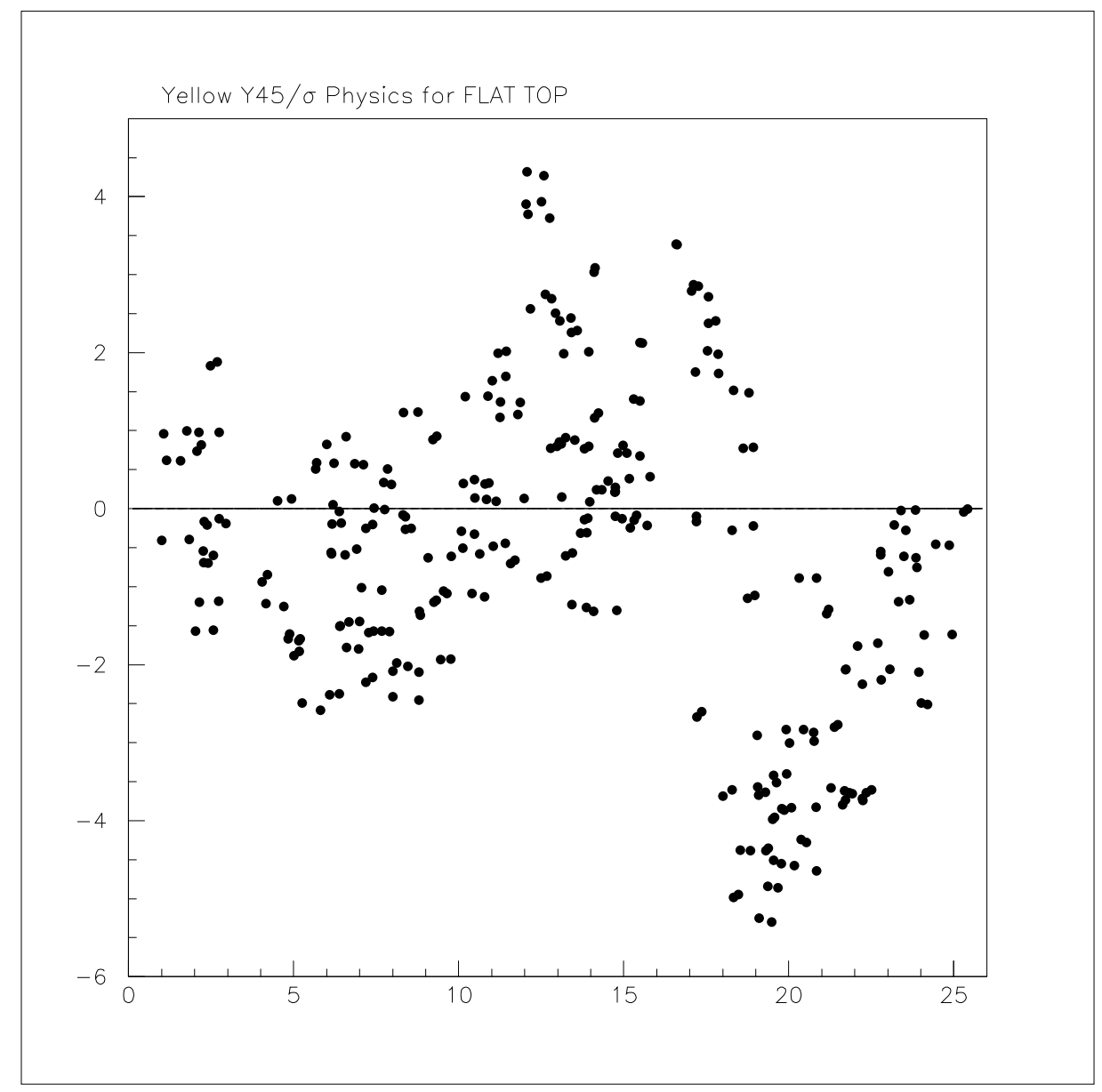

FIG. 10: $\epsilon_{Y 45} / \sigma_{Y 45}$ in the Yellow ring after acceleration to flat top. The horizontal axis is in days in January 2002.

those with hardware problems or special conditions) are shown in Fig. 11. Note data from both rings are combined. If the RHIC polarimeters operated with negligible systematic errors, the means of the two distributions should be zero and the standard deviations should be 1.00. However, the means of $\epsilon_{c r 45} / \sigma_{c r 45}$ and $\epsilon_{Y 45} / \sigma_{Y 45}$ were -0.58 and -0.05 for the Blue and -0.21 and -0.11 for the Yellow beam, while the standard deviations were 2.09 and 1.51 for the Blue and 1.56 and 1.78 for the Yellow beam, respectively. These results suggest that systematic errors are comparable in magnitude to statistical uncertainties, or perhaps as large as $\sqrt{(2.09)^{2}-1.0}=1.83$ times this magnitude. Note this is close to the estimate made from the luminosity asymmetries. 

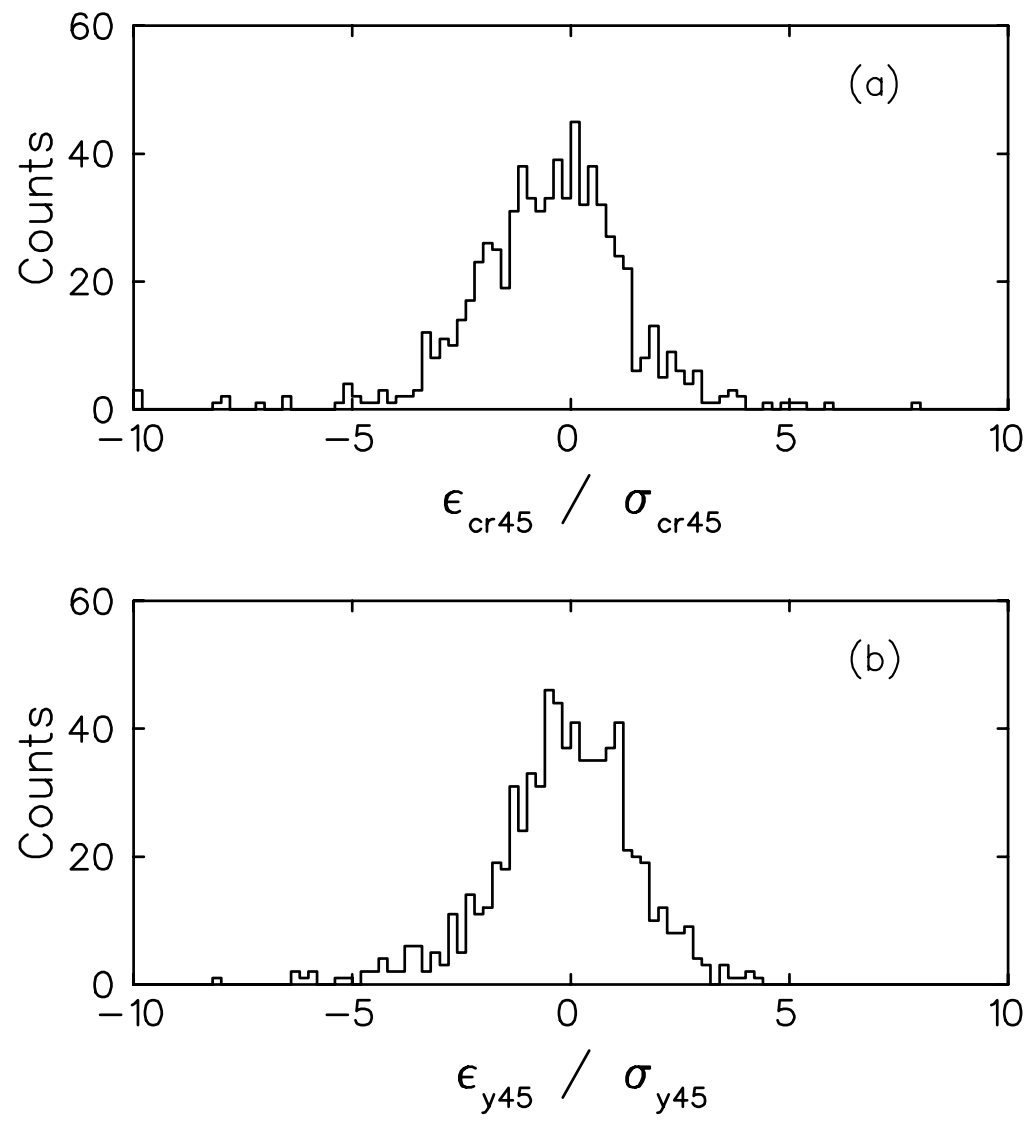

FIG. 11: Histograms of the ratios of value to statistical uncertainty for (a) $\epsilon_{c r 45}$ and (b) $\epsilon_{Y 45}$. Both of these quantities should average to zero and have a width of 1.0.

The variation in $\epsilon_{Y 45}$ appears to be somewhat correlated with the maximum difference in luminosity asymmetries, $\Delta \epsilon_{M A X}$. Figure 12 shows the Yellow ring values for $\Delta \epsilon_{M A X}$ divided by the corresponding statistical errors versus day for all flattop measurements in the same time period as Fig. 10. (The luminosity asymmetry difference with the maximum magnitude among $\epsilon_{2-5}-\epsilon_{1-4}, \epsilon_{2-5}-\epsilon_{3-6}$, and $\epsilon_{3-6}-\epsilon_{1-4}$ is defined to be $\Delta \epsilon_{M A X}$.) The plot of $\epsilon_{Y 45} / \sigma_{Y 45}$ versus $\Delta \epsilon_{M A X} / \sigma_{M A X}$ is presented in Fig. 13, where a weak correlation is seen. The cause of this correlation is not presently understood, but it suggests the presence of a systematic effect in the data.

Based on work by Joanna Kiryluk looking at the STAR beam-beam counters, it was observed that some RHIC fills had $\sim 5$ adjacent bunches with anomalous behaviour in specific luminosity compared to the remainder of the bunches. There were 11 fills identified that exhibited this behavior (2233, 2235, 2244, 2246, 2251, 2266, 2269, 2275, 2289, 2301, 


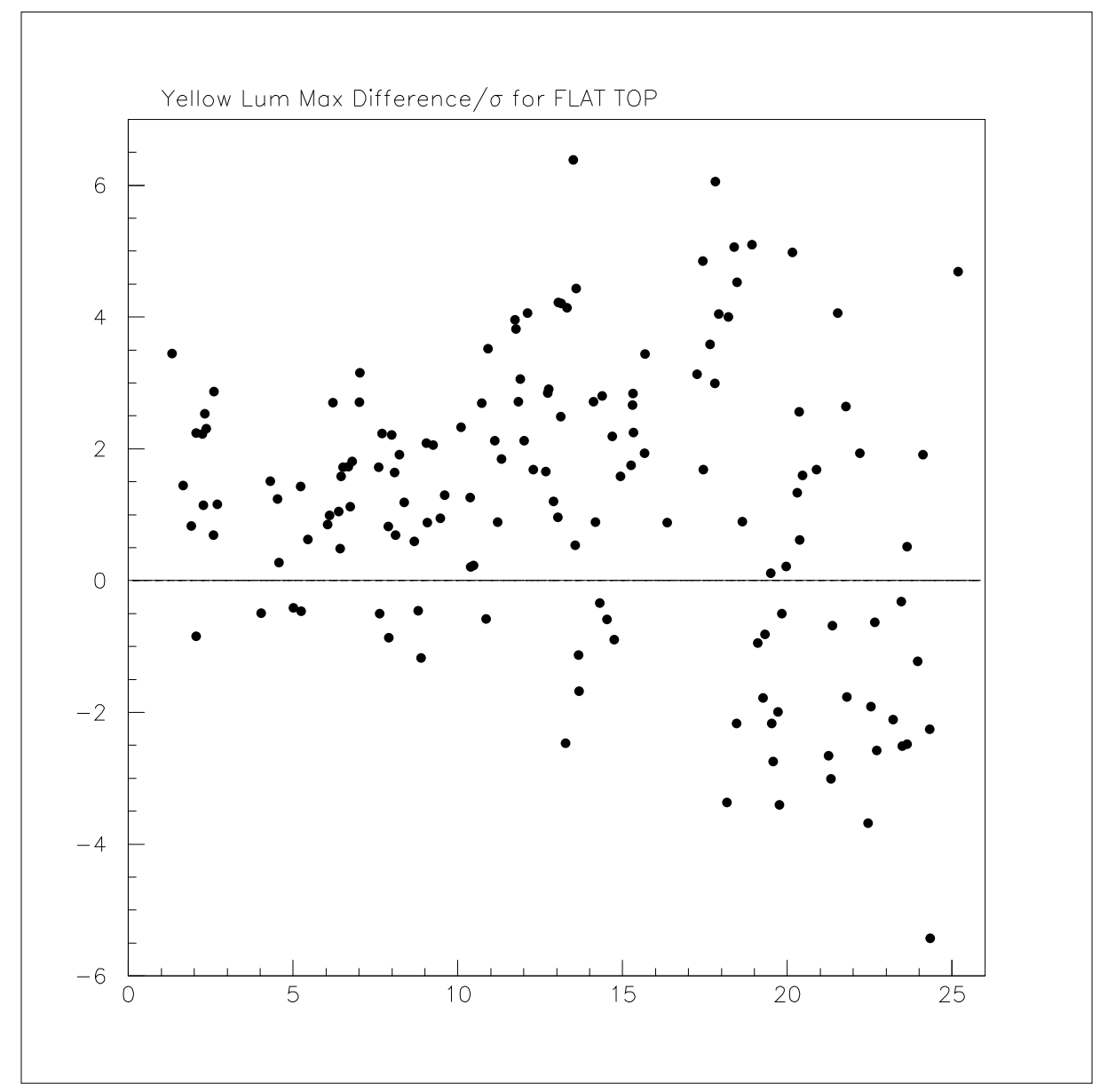

FIG. 12: $\Delta \epsilon_{M A X} / \sigma_{M A X}$ vs. day for all flat top measurements in the Yellow ring in January 2002.

2303). The number of runs with sizable differences in $\epsilon_{\text {lum }}$ compared to the total was $11 / 57=0.193 \pm 0.021$ for the Blue and $15 / 47=0.319 \pm 0.032$ for the Yellow ring in these fills. However, there were sizable differences during 10/42 $=0.238 \pm 0.028$ runs for the Blue and $15 / 36=0.417 \pm 0.041$ for the Yellow ring for nominally "good fills" between 2233 and 2303. The fractions are nearly equal for good and bad fills, with perhaps the good fills having a slightly higher rate of large $\epsilon_{\text {lum }}$ differences. In particular, the anomalous behaviour of the 5 adjacent bunches in some fills does not seem to be strongly correlated with sizeable $\epsilon_{l u m}$ differences. 


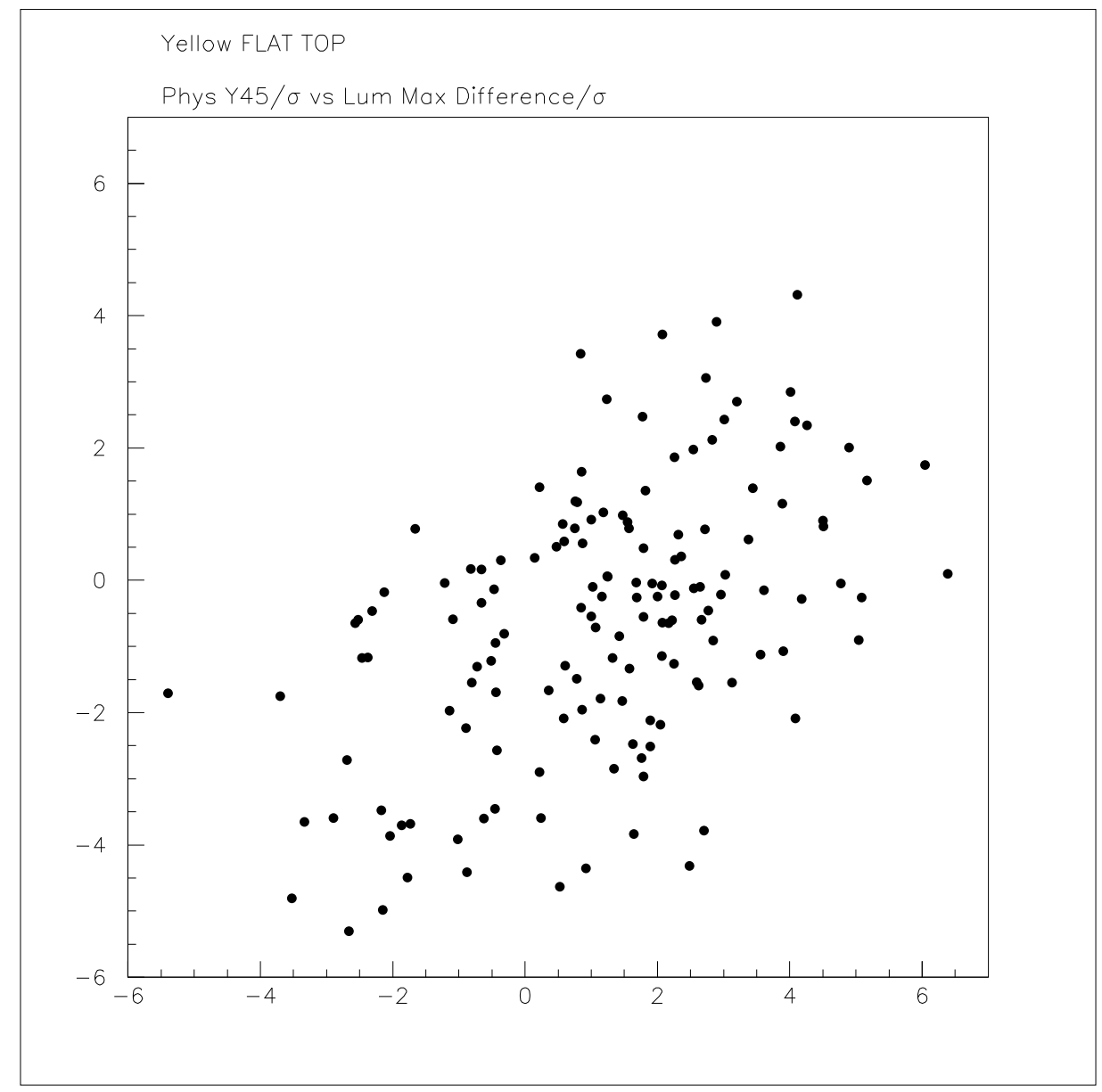

FIG. 13: $\epsilon_{Y 45} / \sigma_{Y 45}$ vs. $\Delta \epsilon_{M A X} / \sigma_{M A X}$ for all flat top measurements in the Yellow ring in January 2002.

\section{Other Correlations}

In the absence of sizeable systematic effects, there should be no correlations of the physics asymmetry with either the luminosity or geometric asymmetry. This was tested with a subset of the good runs from mid January 2002. The Blue ring results for the detectors in the horizontal plane (X90) are shown in Fig. 14. No correlation is seen with the luminosity asymmetry, but there is a weak correlation with the geometrical asymmetry. The same behavior is also seen for the Yellow ring (not shown). If the correlation is real, this would signal the presence of systematic effects. However, it is also possible that there were periods when the beam polarization was high and when the geometrical asymmetry happened to 

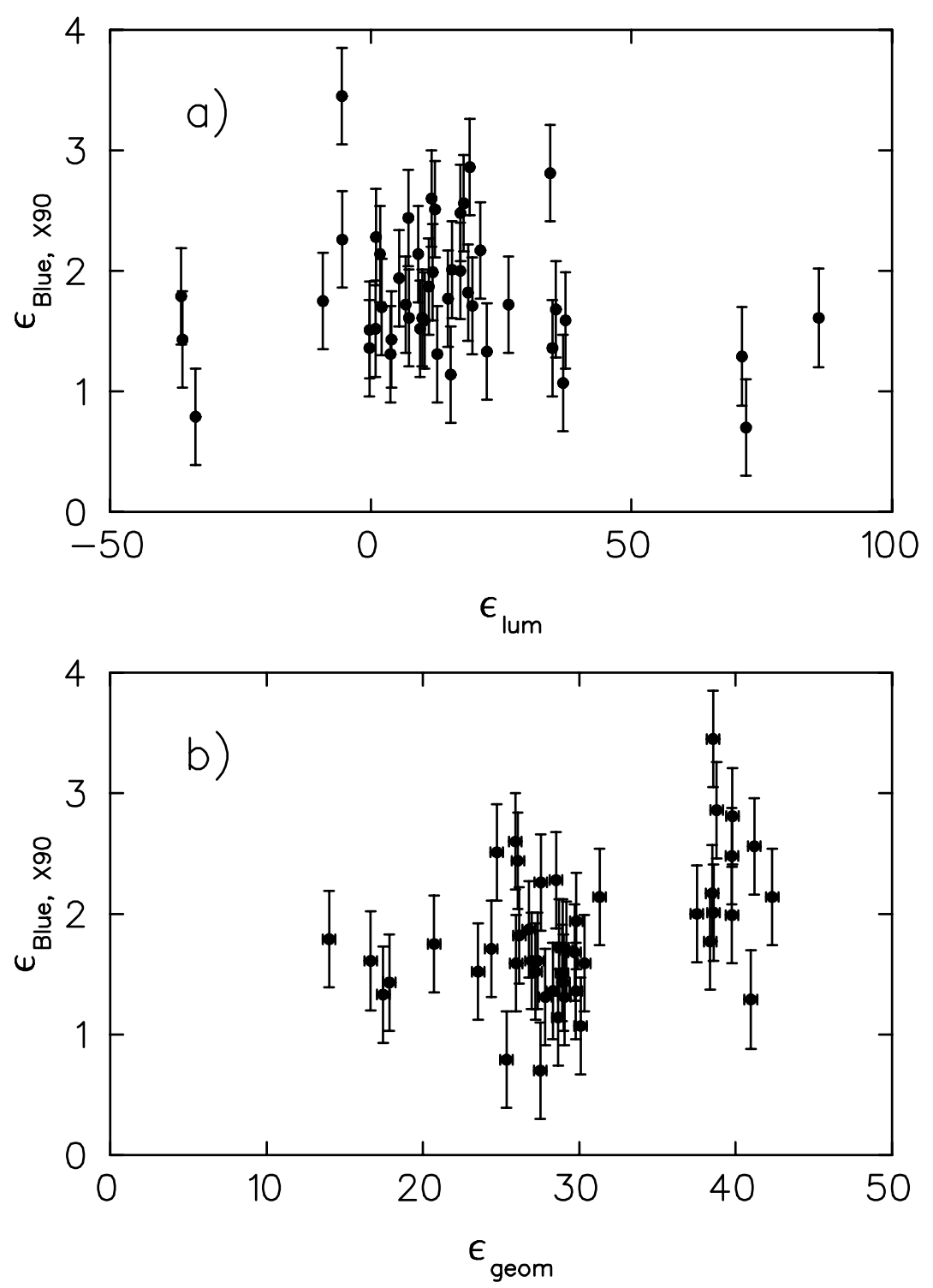

FIG. 14: Correlation of the X90 physics asymmetry for the Blue ring with a) the X90 luminosity asymmetry. b) the X90 geometrical asymmetry. All asymmetries are multiplied by 1000 .

be high as well, as the data shown were collected over a four day period. Note that the correlation with $\epsilon_{\text {geom }}$ has the same sort of behavior seen in Fig. 1 for the 200-MeV polarimter.

The data from detectors $1,3,4$, and 6 at $\pm 45^{\circ}$ to the horizontal plane were summed into "left" $(1,3)$ and "right" $(4,6)$, and the physics asymmetry from Eq. 6 was denoted $\epsilon_{X 45}$. This asymmetry should be equal to the physics asymmetry from detectors 2 and $5, \epsilon_{X 90}$. The correlation of these two asymmetries is shown in Fig. 15 for the Blue and Yellow rings. 

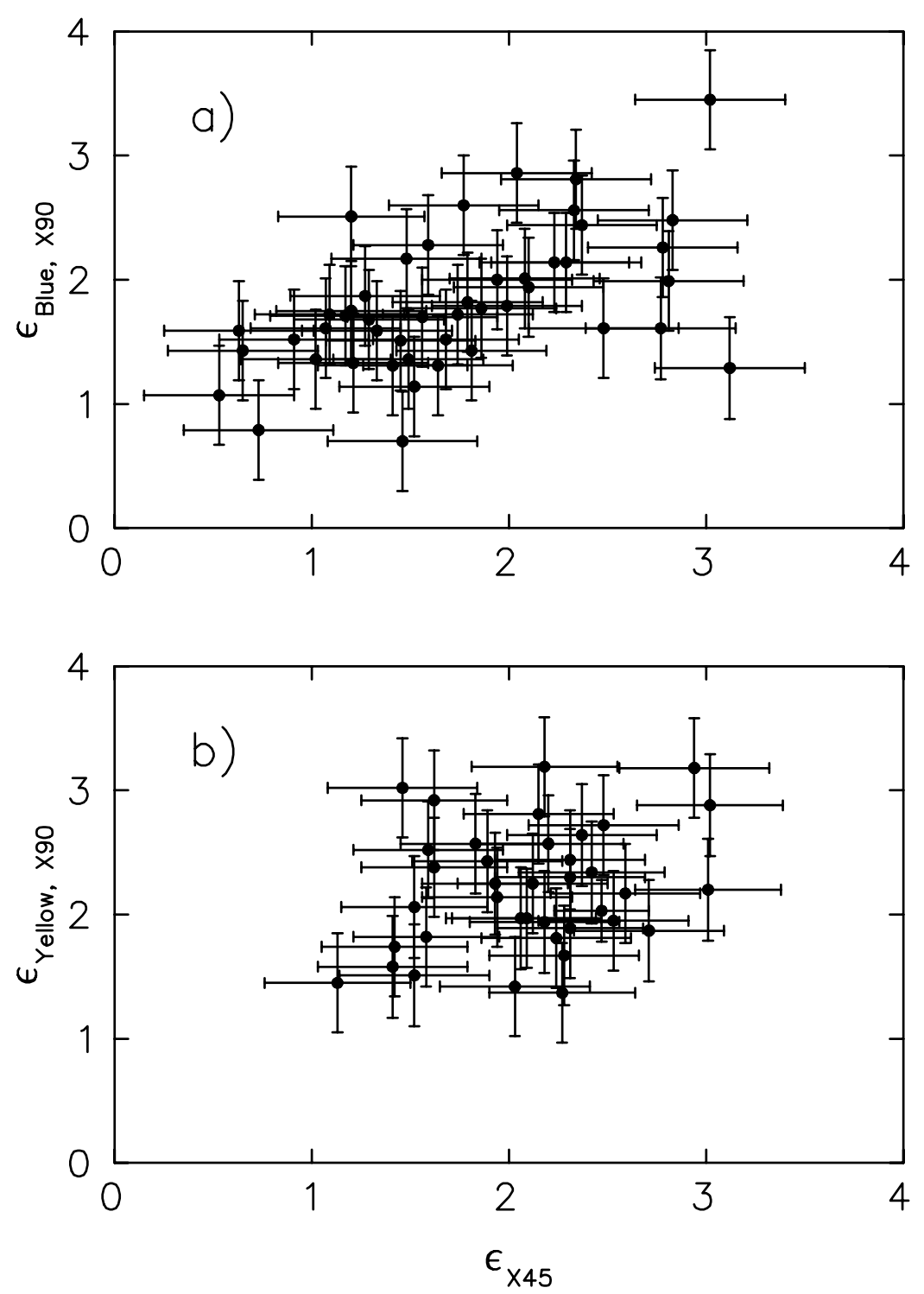

FIG. 15: Correlation of the X90 with the X45 physics asymmetries. These are expected to be equal. a) Blue ring. b) Yellow ring. All asymmetries are multiplied by 1000 .

The data cluster around the $\epsilon_{X 90}=\epsilon_{X 45}$ line.

The ratio of physics asymmetries, $\epsilon_{X 90} / \epsilon_{X 45}$, was computed for all runs without hardware problems, and gave a weighted average $0.819 \pm 0.015\left(\chi^{2} / d . f .=719.7 / 389\right)$ and $0.867 \pm 0.014$ $\left(\chi^{2} /\right.$ d.f. $\left.=356.4 / 333\right)$ for the Blue and Yellow ring polarimeters, respectively. Restricting the data to those after run 600 (10 January 2002) gave $0.982 \pm 0.023$ (185.5/163 d.f.) and $0.898 \pm 0.019$ (177.6/154 d.f.). The later Blue ring data are consistent with 1.00, as expected, 
but the Yellow ring results suggest a possible calibration difference between $\epsilon_{X 90}$ and $\epsilon_{X 45}$, perhaps due to details of which silicon strips were excluded from the data or from the thickness of the dead layers on the strips. The fact that the $\chi^{2} / d$. $f$. for the later runs are close to 1.00 is encouraging, suggesting the systematic errors in the polarimeter data may be somewhat smaller than the statistical uncertainties.

The variation of $\epsilon_{X 90}$ relative to $\epsilon_{X 45}$ outside statistics could be related to changes in the average beam position for + and - bunches. It was shown in Sec. IV.A that there is no effect on these asymmetries for the same offset for both type bunches (fixed $d \Omega_{L}, d \Omega_{R}$ ). Since the CNI polarimeter target is a thin, vertical "ribbon", consider the case where the + bunches are systematically high by $\delta y$ and the - bunches are systematically low by $\delta y$. As the detectors are much wider than the desired elastic scattering events from the target, the major effect on the solid angles will be the change in the $\phi$ acceptances. An overall offset of the beam from the nominal beam height, $\Delta$, will also be considered.

For the detectors in the horizontal plane, 2 and 5 ,

$$
\begin{aligned}
& d \Omega_{L}^{\uparrow} \propto \frac{1}{\sqrt{\left(R_{2}\right)^{2}+(\Delta+\delta y)^{2}}} \\
& d \Omega_{L}^{\downarrow} \propto \frac{1}{\sqrt{\left(R_{2}\right)^{2}+(\Delta-\delta y)^{2}}} \\
& d \Omega_{R}^{\uparrow} \propto \frac{1}{\sqrt{\left(R_{5}\right)^{2}+(\Delta+\delta y)^{2}}} \\
& d \Omega_{R}^{\downarrow} \propto \frac{1}{\sqrt{\left(R_{5}\right)^{2}+(\Delta-\delta y)^{2}}}
\end{aligned}
$$

and the effects on the physics and luminosity asymmetries would be

$$
\begin{aligned}
\epsilon_{\text {phys }} & \rightarrow P A+\left(\frac{\delta y}{R}\right) \cdot\left(\frac{\Delta}{R}\right) \cdot\left(\frac{R_{2}-R_{5}}{R}\right)+\text { h.o.t. } \\
\epsilon_{\text {lum }} & \rightarrow \epsilon_{B}+P A \epsilon_{A}-\left(\frac{\delta y}{R}\right) \cdot\left(\frac{\Delta}{R}\right)+\text { h.o.t. }
\end{aligned}
$$

In order to make estimates of the sizes of these corrections, the offset between the + and bunches will be assumed to be $\delta y \sim 0.15 \mathrm{~mm}$, the overall offset of the beam $\Delta \sim 1.5 \mathrm{~mm}$, and the difference in distances $\left(R_{2}-R_{5}\right) \sim 1.5 \mathrm{~mm}$. The average distance from the target to the RHIC CNI polarimeter detectors is $R=\left(R_{2}+R_{5}\right) / 2=15 \mathrm{~cm}$. Then the effects on $\epsilon_{\text {phys }}$ and $\epsilon_{\text {lum }}$ would be of order $10^{-7}$ and $10^{-5}$, respectively, which are negligible compared to 
the statistical uncertainties $\left(\sim 4 \times 10^{-4}\right)$. Thus, to a good approximation, the polarimeter is insensitive to differences in vertical beam position for + and - bunches for the $2-5$ detector pair.

For the $\pm 45^{\circ}$ detectors $(1,3,4,6)$ the equations are somewhat more complicated. It will be assumed that $d \Omega_{L}$ is the simple, unweighted sum of solid angles for detectors 1 and 3 , and $d \Omega_{R}$ is the sum of solid angles for detectors 4 and 6 . Then

$$
\begin{aligned}
& d \Omega_{L}^{\uparrow} \propto \frac{1}{\sqrt{\left(R_{1}-\frac{\Delta+\delta y}{\sqrt{2}}\right)^{2}+\left(\frac{\Delta+\delta y}{\sqrt{2}}\right)^{2}}}+\frac{1}{\sqrt{\left(R_{3}+\frac{\Delta+\delta y}{\sqrt{2}}\right)^{2}+\left(\frac{\Delta+\delta y}{\sqrt{2}}\right)^{2}}} \\
& d \Omega_{L}^{\downarrow} \propto \frac{1}{\sqrt{\left(R_{1}-\frac{\Delta-\delta y}{\sqrt{2}}\right)^{2}+\left(\frac{\Delta-\delta y}{\sqrt{2}}\right)^{2}}}+\frac{1}{\sqrt{\left(R_{3}+\frac{\Delta-\delta y}{\sqrt{2}}\right)^{2}+\left(\frac{\Delta-\delta y}{\sqrt{2}}\right)^{2}}} \\
& d \Omega_{R}^{\uparrow} \propto \frac{1}{\sqrt{\left(R_{4}+\frac{\Delta+\delta y}{\sqrt{2}}\right)^{2}+\left(\frac{\Delta+\delta y}{\sqrt{2}}\right)^{2}}}+\frac{1}{\sqrt{\left(R_{6}-\frac{\Delta+\delta y}{\sqrt{2}}\right)^{2}+\left(\frac{\Delta+\delta y}{\sqrt{2}}\right)^{2}}} \\
& d \Omega_{R}^{\downarrow} \propto \frac{1}{\sqrt{\left(R_{4}+\frac{\Delta-\delta y}{\sqrt{2}}\right)^{2}+\left(\frac{\Delta-\delta y}{\sqrt{2}}\right)^{2}}}+\frac{1}{\sqrt{\left(R_{6}-\frac{\Delta-\delta y}{\sqrt{2}}\right)^{2}+\left(\frac{\Delta-\delta y}{\sqrt{2}}\right)^{2}}}
\end{aligned}
$$

The effects on the physics and luminosity asymmetries would be

$$
\begin{aligned}
\epsilon_{\text {phys }} \rightarrow & P A+\frac{1}{2 \sqrt{2}} \cdot\left(\frac{\delta y}{R}\right) \cdot\left[\frac{\left(R_{3}-R_{1}\right)-\left(R_{4}-R_{6}\right)}{R}\right]+ \\
& \left(\frac{\Delta}{R}\right) \cdot\left(\frac{\delta y}{R}\right) \cdot\left[\frac{\left(R_{3}+R_{1}\right)-\left(R_{4}+R_{6}\right)}{R}\right]+\text { h.o.t. } \\
\epsilon_{\text {lum }} \rightarrow & \epsilon_{B}+P A \epsilon_{A}+\frac{1}{2 \sqrt{2}} \cdot\left(\frac{\delta y}{R}\right) \cdot\left[\frac{\left(R_{3}-R_{1}\right)+\left(R_{4}-R_{6}\right)}{R}\right]- \\
& \left(\frac{\Delta}{R}\right) \cdot\left(\frac{\delta y}{R}\right)+\text { h.o.t. }
\end{aligned}
$$

In this case, the magnitudes of the effects are of order $10^{-5}$ (assuming the same sizes for $\Delta$, $\delta y$, and distances to the detectors as before); these are small compared to statistical uncertainties. In conclusion, the physics and luminosity asymmetries for the RHIC polarimeters (both X45 and X90) are insensitive to differences in vertical beam position for + and bunches. Hence differences observed between $\epsilon_{X 45}$ and $\epsilon_{X 90}$ or between luminosity asymmetries are not believed to originate with changes in vertical beam position correlated with spin state. 


\section{RHIC POLARIMETER AND RHIC PERFORMANCE}

\section{A. RHIC Injection Asymmetries}

It was expected that the average Blue and Yellow ring polarizations at injection would be approximately the same. A small difference might have been present due to the AGS partial snake and interspersed vertical and horizontal bends in the transfer line to RHIC (Ref. [9]). However, significant departures from equality might suggest problems in the operation of the AGS, the AGS to RHIC transfer line, RHIC, or the polarimeters. In the ideal case of equal polarizations in the two rings, the ratio of polarimeter asymmetries at injection for the Yellow and Blue rings should be the same as the ratio of analyzing powers:

$$
\frac{\epsilon_{\text {inj.Yellow }}}{\epsilon_{\text {inj. Blue }}}=\frac{A_{\text {Yellow }}}{A_{\text {Blue }}}=\frac{0.0133 \pm 0.0015}{0.0127 \pm 0.0011}=1.05 \pm 0.15,
$$

where the RHIC polarimeter analyzing powers are the best estimates as of the time of this note. The ratio was formed for 41 fills from data in version 1.1 of the RHIC polarimeter spreadsheet. It was required that no known hardware problems were present and that the magnitude of each asymmetry had to be larger than $1.0 \times 10^{-3}$. The ratio is plotted as a function of time in Fig. 16. Figure 17 includes histograms of individual Blue and Yellow ring polarimeter asymmetries and their correlation.

Three types of asymmetries were used to compute the ratio: 1) $\left.\epsilon_{2-5}=\epsilon_{X 90}, 2\right) \epsilon_{X 45}$, and 3) the least squares fit $\mathrm{X}$ asymmetry. For $\epsilon_{X 45}$, the counts from detectors 1 and 3 were summed and from 4 and 6 were summed to give the equivalent of "left - right detectors." The least squares fit asymmetries used information from all six detectors to derive a best estimate of the left-right $(\mathrm{X})$ and up-down $(\mathrm{Y})$ asymmetries. The mean values for the three types of asymmetry ratios are $0.918 \pm 0.033,1.038 \pm 0.032$, and $1.024 \pm 0.023$, respectively, and the reduced chi-squares are 1.575, 2.002, and 2.078. There is good agreement of the means with Eq. 12 for all three types of asymmetries, but the X90 result seems to differ from the other two. Since most of the data had the Blue ring filled before the Yellow ring, any significant depolarization in RHIC at the injection energy should have decreased $\epsilon_{\text {inj.Blue }}$ and increased the ratio above that expected in Eq. 12. Similarly, the small difference in polarization predicted in Ref. [9] was expected to increase the ratio. Yet all three ratios are below 1.05, suggesting such effects are small.

The sizable reduced chi squares are of concern, and may suggest one or more of the 

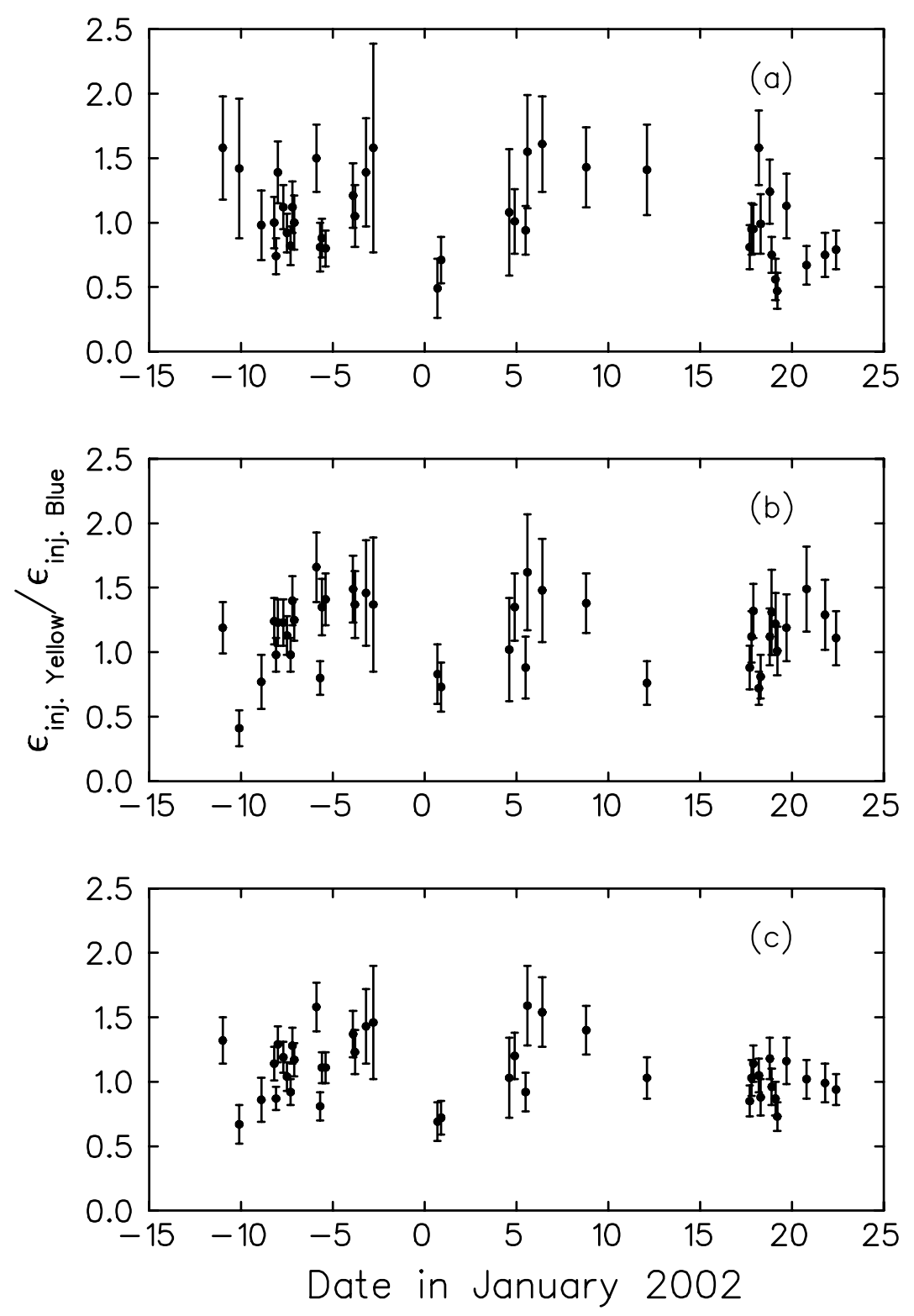

FIG. 16: Time dependence of the ratio of Yellow ring to Blue ring asymmetry at injection for three types of asymmetries. a) $\epsilon_{X 90}$, b) $\epsilon_{X 45}$, and c) the least squares fit $\mathrm{X}$ asymmetry.

problems noted above. If the cause is purely from systematic error in the polarimeters, then this error is of comparable magnitude to the statistical uncertainty, which is consistent with the estimates from luminosity asymmetry differences, as well as $\epsilon_{c r 45}$ and $\epsilon_{Y 45}$. Thus, there is no strong evidence for problems with the operations of the AGS, AGS to RHIC transfer line, or RHIC on the basis of the ratio of asymmetries in the two rings at injection. 


\section{RHIC polarized pp performance (injection)}
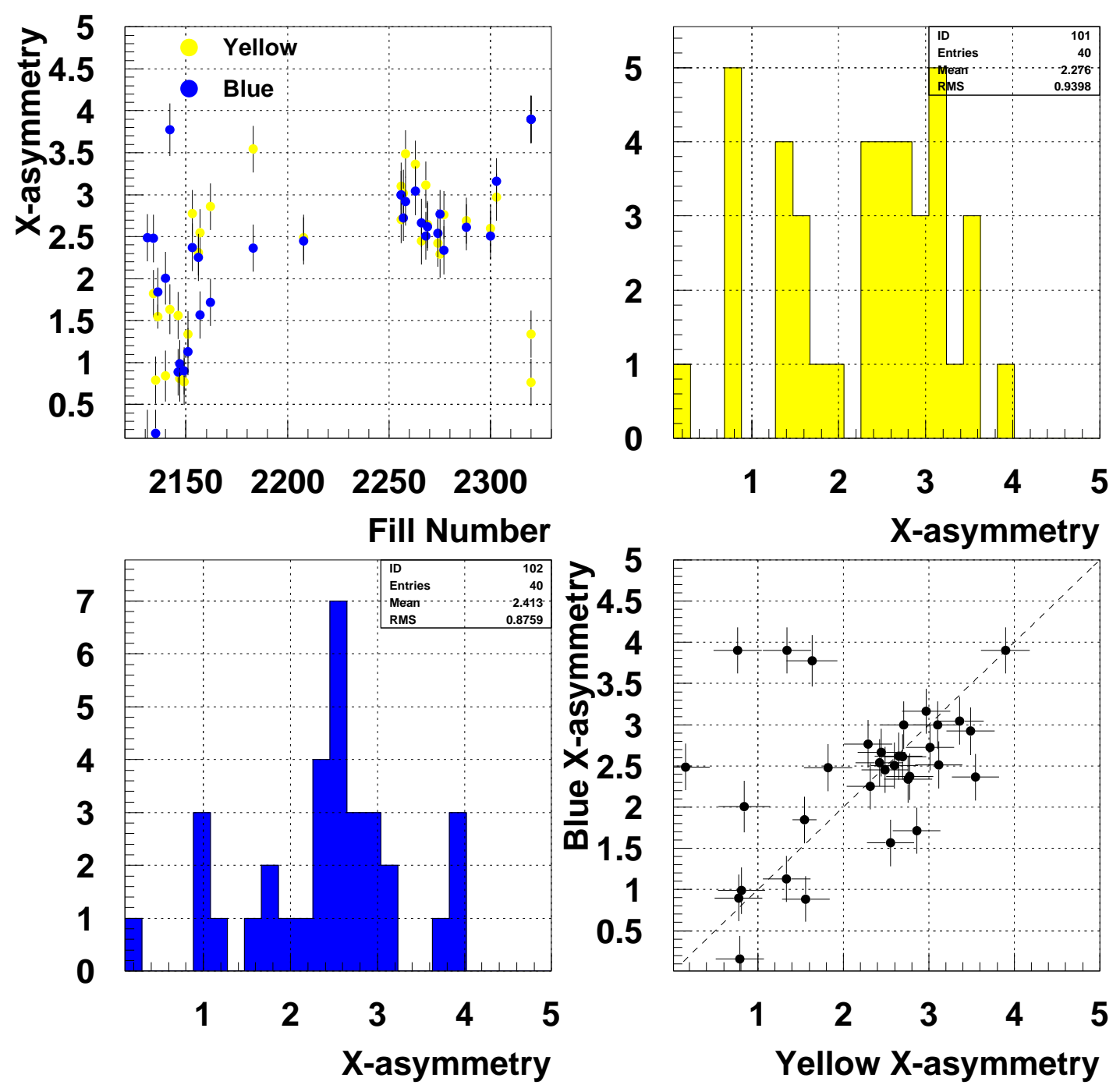

FIG. 17: Histograms of least squares fit $\mathrm{X}$ asymmetries (multiplied by 1000) from the RHIC polarimeters at injection. The upper left figure is the asymmetry as a function of fill number (time). The upper right and lower left correspond to the Yellow and Blue asymmetries, respectively, and the lower right shows the correlation.

\section{B. Analyzing Power at Flattop}

An attempt was made to determine a lower limit to the analyzing power of the polarimeters at flattop $(100 \mathrm{GeV})$. It was assumed that the analyzing powers at injection $\left(A_{i n j}\right)$ and 
flattop $\left(A_{\text {flat }}\right)$ were constant with time; hardware changes were assumed to negligibly affect the values of $A_{i n j}$ or $A_{\text {flat }}$. The measured asymmetries were

$$
\begin{aligned}
\epsilon_{\text {flat }} & =P_{\text {flat }} \times A_{\text {flat }} \\
\epsilon_{i n j} & =P_{i n j} \times A_{i n j},
\end{aligned}
$$

where $P_{i n j}$ and $P_{\text {flat }}$ were the beam polarizations. From these equations,

$$
\frac{A_{f l a t}}{A_{i n j}}=\frac{\epsilon_{f l a t}}{\epsilon_{i n j}} \times \frac{P_{i n j}}{P_{f l a t}} \geq \frac{\epsilon_{f l a t}}{\epsilon_{i n j}},
$$

since depolarization may have occurred between injection and flattop. Histograms of the asymmetry ratio, $\epsilon_{f l a t} / \epsilon_{i n j}$, were made to estimate the largest value achieved, and thus the best lower limit on $A_{f l a t} / A_{i n j}$. Note that it is possible that sizable depolarization occurred between injection and flattop for all runs, so that the derived limit on $A_{\text {flat }} / A_{\text {inj }}$ could be significantly below the true value.

Fills were selected with at least one asymmetry measurement at injection and one at flattop. The ratio $\epsilon_{f l a t} / \epsilon_{i n j}$ was computed with asymmetries from the same ring whenever possible. However, there were a substantial number of cases where $\epsilon_{i n j}$ was only measured in the Blue ring, and several only in the Yellow ring. For these cases, the injection asymmetry in the two rings was assumed to be equal, so that the $\epsilon_{f l a t} / \epsilon_{i n j}$ ratio could be computed for the ring without an asymmetry measurement at injection. A plot of the asymmetry as a function of time in the Blue ring is given in Fig. 18, with injection data represented differently from flattop results.

A weighted average of up to three measurements on flattop was used to obtain $\epsilon_{\text {flat }}$. These were nearly always the first three flattop asymmetries measured in that ring. For very long runs with many flattop measurements, there was evidence for a drop in asymmetry with time; see Sec. V.C. Runs with evidence for hardware problems or with special operating conditions were excluded. Histograms of the ratio for the various combinations of rings are shown in Fig. 19, and the sum is given in Fig. 20. The four largest ratios are $2.01 \pm 1.17$, $1.63 \pm 0.26,1.40 \pm 0.53$, and $1.28 \pm 0.24$. The dashed line in Fig. 20 corresponds to all data, including the smaller ratio from fills where $\epsilon_{\text {flat }}$ from the two rings differed by more than three standard deviations, while the solid line in Fig. 20 rejects the smaller ratio in this case.

No statistical procedure has been found (yet) that gives an unbiased estimate for the maximum value of the distributions in Figs. 19 and 20. Therefore, an estimate was made 
RHIC polarized pp performance (01/01/02 - 01/25/02): Blue Beam

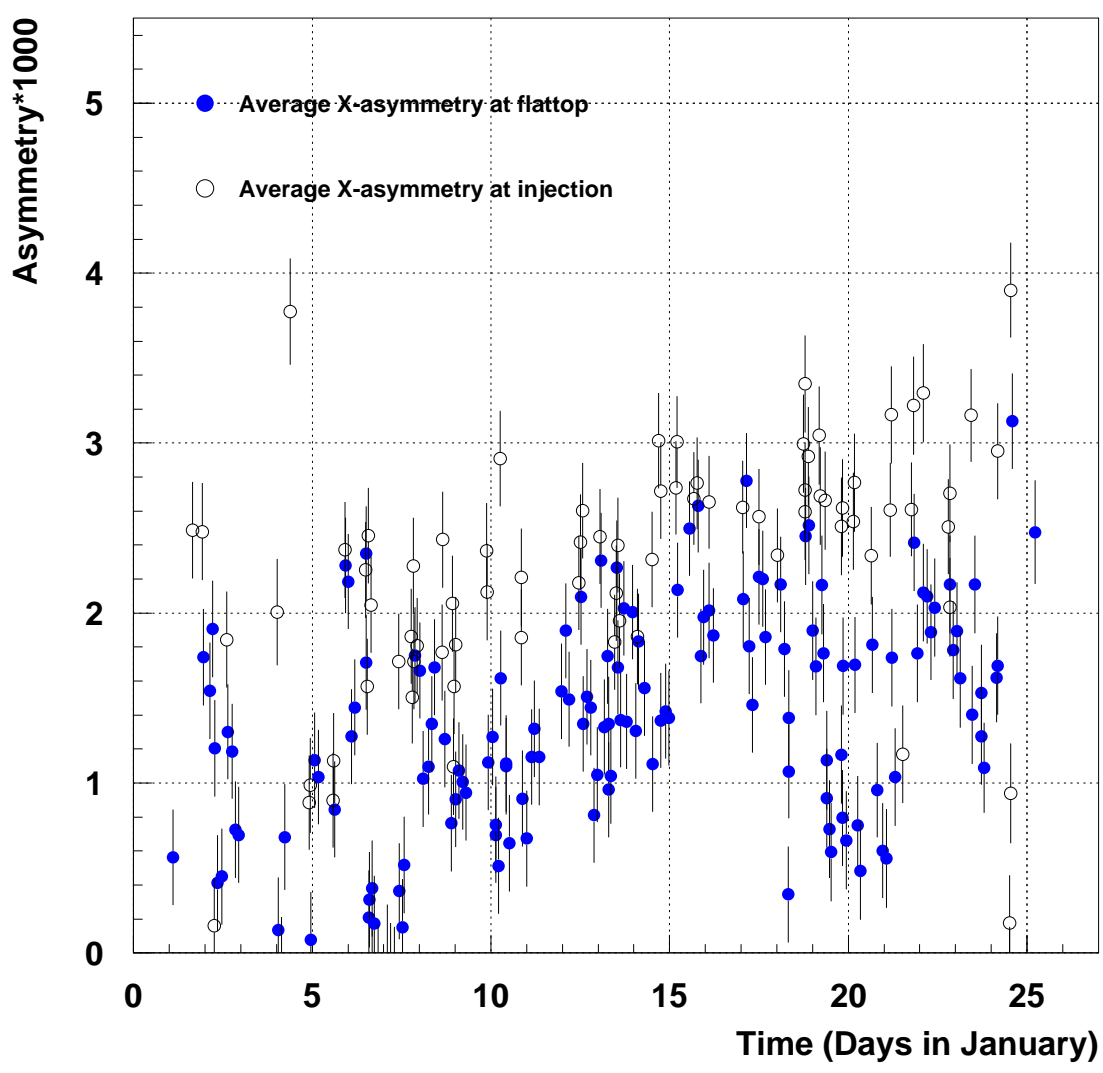

FIG. 18: Least squares fit X asymmetries for the Blue ring RHIC polarimeter as a function of time. Since the injection asymmetries are generally larger than those at flattop, and since the analyzing power at flattop is equal to or larger than at injection, these results indicate sizable polarization loss between injection and flattop.

taking into account the statistical errors of the largest ratios, the shape of the distributions, and the total number of ratios. A conservative maximum value appears to be $\epsilon_{\text {flat }} / \epsilon_{i n j} \geq 1.0$, which then gives

$$
A_{\text {flat }} / A_{i n j} \geq 1.0
$$

This result is consistent with that assumed during the recent RHIC polarized proton runs $\left(A_{\text {flat }} / A_{\text {inj }}=1.0\right)$. 

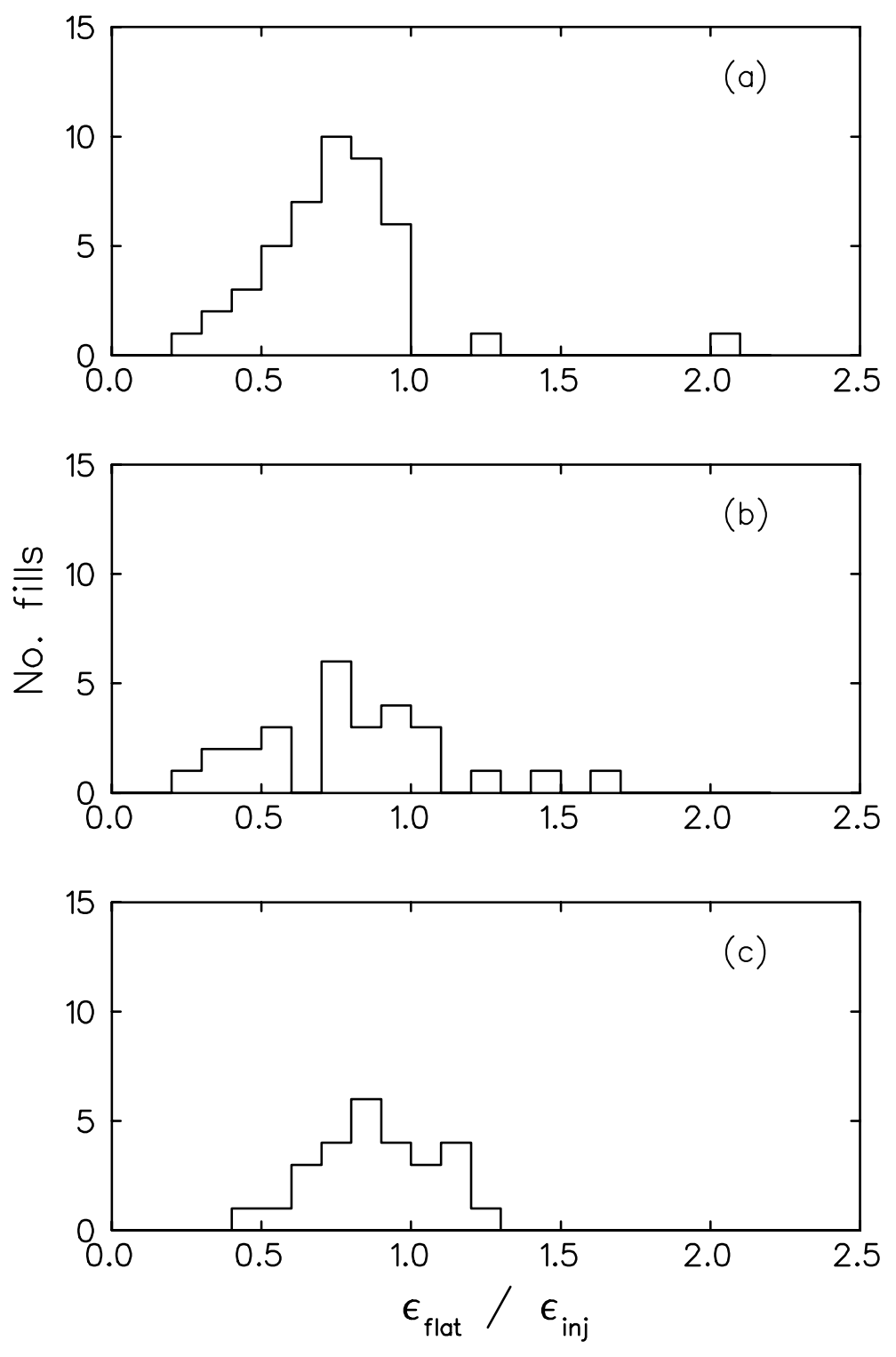

FIG. 19: Histogram of the ratio of asymmetries at flattop to injection. a) Blue ring data for both flattop and injection. b) Yellow ring data for both flattop and injection. c) Results for one ring at injection (usually Blue) and the other at flattop.

\section{Beam Polarization on Flattop}

The RHIC snakes are expected to prevent beam depolarization during the flattop. However, the data often show a drop in asymmetry with time in the fill; see Figs. 21 and 22. This expectation was tested with the polarimeter data by forming asymmetry ratios to the 

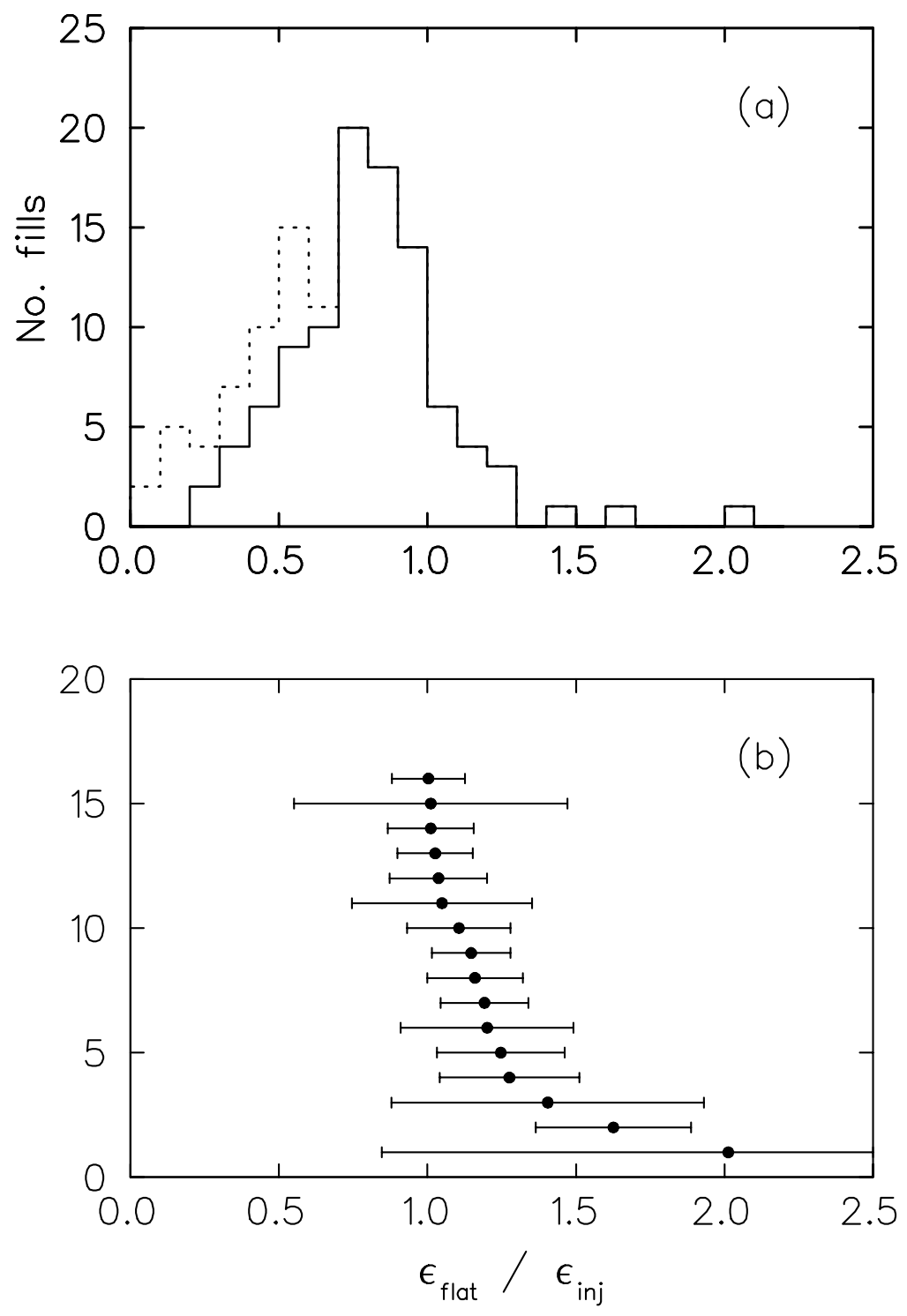

FIG. 20: a) Histogram of the ratio of asymmetries at flattop to injection from the three groups shown in Fig. 19. The dashed line contains all ratios, while the solid line rejects results with small $\epsilon_{\text {flat }}$ as described in the text. b) Individual measurements of $\epsilon_{f l a t} / \epsilon_{i n j}$ shown with statistical errors, ordered by size. All results with a ratio greater than or equal to 1.0 are shown.

first measurement on flattop:

$$
\text { Ratio }=\epsilon_{j} / \epsilon_{1}, \quad 2 \leq j \leq n \text {. }
$$

Each ratio had both asymmetries measured in the same fill and beam. Several conditions were applied to the data: 1) At least three polarimeter measurements were required on flattop 
RHIC polarized pp performance (01/01/02 - 01/25/02): Blue Beam

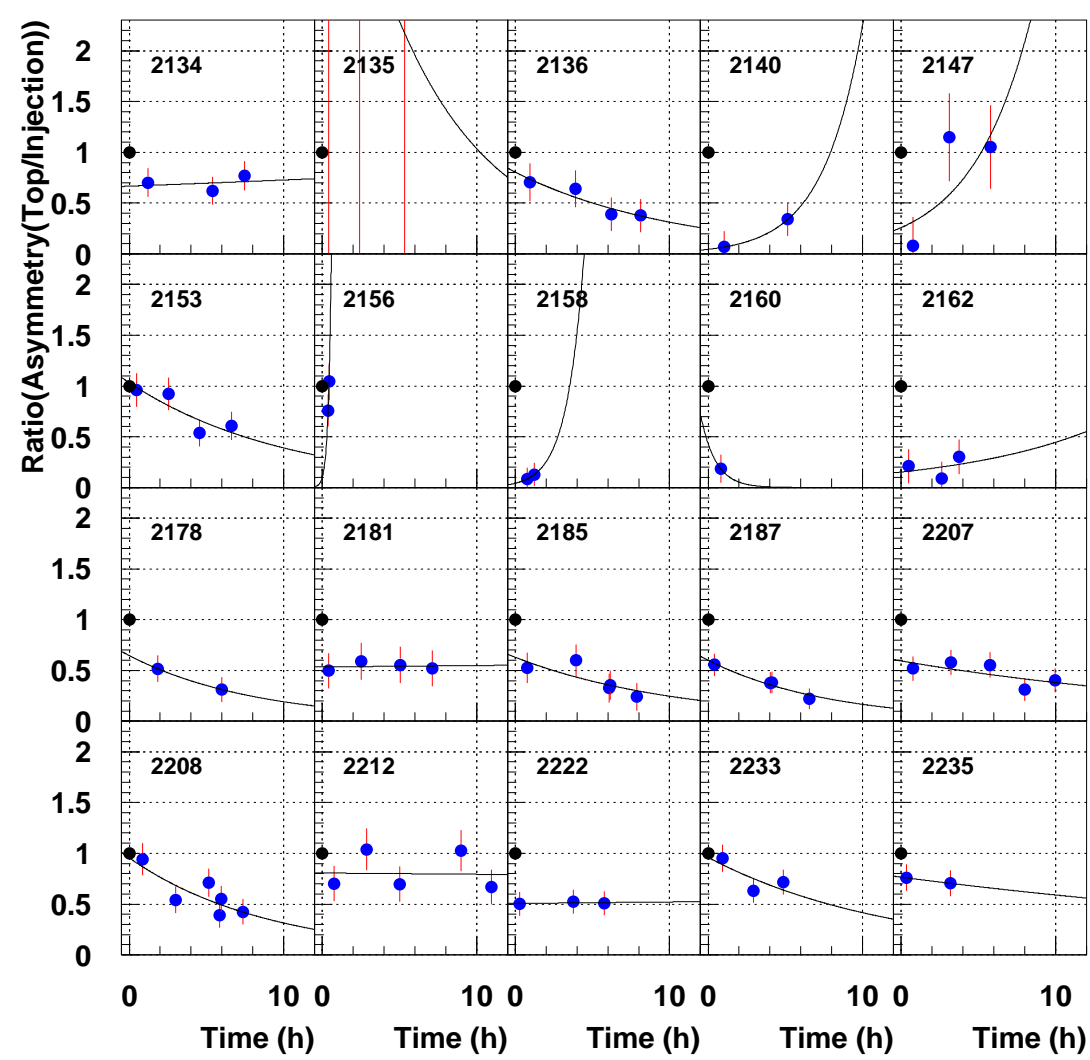

FIG. 21: Plots of $\mathrm{X}$ asymmetries normalized to the asymmetry at injection as a function of time within a fill for the Blue ring RHIC polarimeter. The fill number is noted.

in the same beam (Yellow or Blue) and fill. 2) The magnitude of the initial flattop asymmetry had to exceed $1.0 \times 10^{-3}$. 3) Fills with special conditions or with known polarimeter hardware problems were excluded.

The ratios were placed in one of seven time bins after the first measurement

$$
2 \pm 1 / 2 \mathrm{hr}, 3 \pm 1 / 2 \mathrm{hr}, \ldots ., 7 \pm 1 / 2 \mathrm{hr}, \quad>7.5 \mathrm{hrs}
$$

and weighted averages computed. A total of 51 sets of runs (beam, fill) are included. The weighted averages of the ratios as a function of time for the Yellow and Blue beam separately are shown in Fig. 23a, and for data from both beams combined in Fig. 23b. These data are also given in Table II below. It can be seen that the asymmetry ratios are all less than 
RHIC polarized pp performance (01/01/02 - 01/25/02): Blue Beam

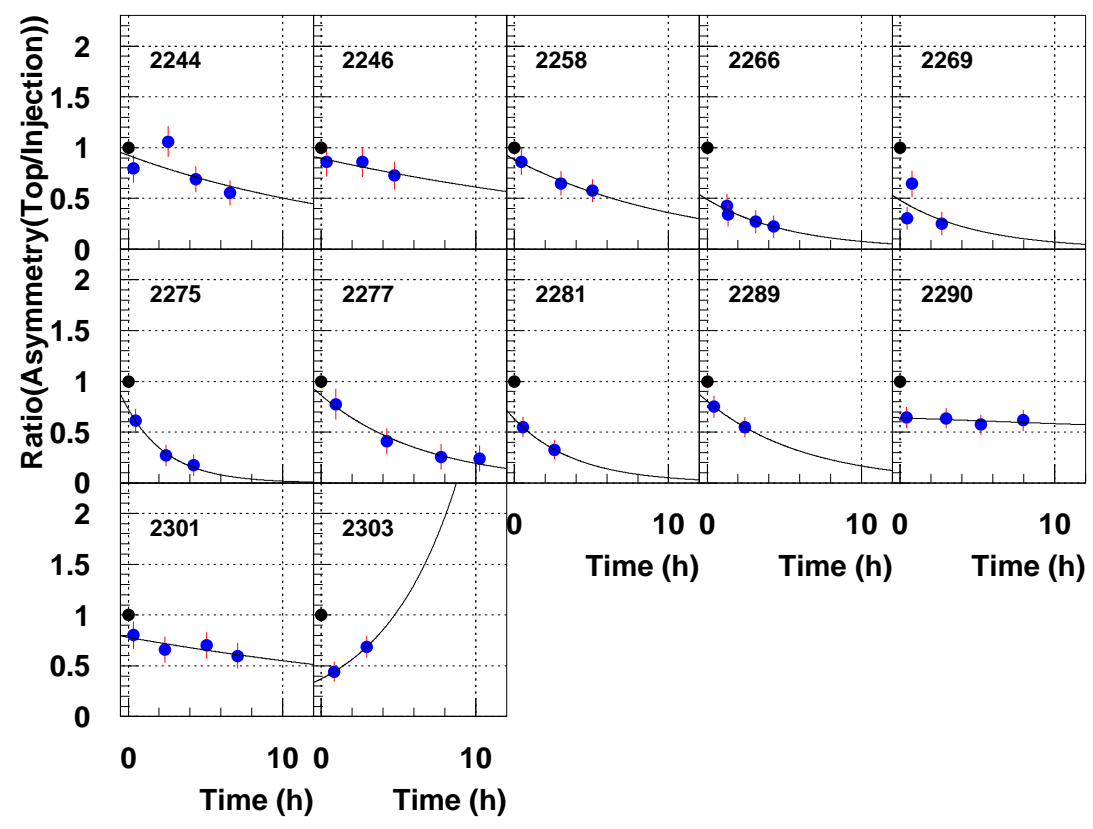

FIG. 22: Plots (cont.) of $\mathrm{X}$ asymmetries normalized to the asymmetry at injection as a function of time within a fill for the Blue ring RHIC polarimeter. The fill number is noted.

TABLE II: Asymmetry ratios on flattop for the Yellow and Blue rings and for the combined results as a function of time from the first measurement on flattop. The reduced $\chi^{2}$ values are shown as well.

\begin{tabular}{|c|c|c|c|c|c|c|}
\hline Time & \multicolumn{2}{|c|}{ Yellow Ring } & \multicolumn{2}{c|}{ Blue Ring } & \multicolumn{2}{c|}{ Combined Data } \\
\hline$(\mathrm{hr})$ & Ratio & $\chi^{2} / \mathrm{ndf}$ & Ratio & $\chi^{2} / \mathrm{ndf}$ & Ratio & $\chi^{2} / \mathrm{ndf}$ \\
\hline 2 & $0.927 \pm 0.041$ & $10.82 / 16$ & $0.832 \pm 0.044$ & $26.38 / 16$ & $0.882 \pm 0.030$ & $39.72 / 33$ \\
3 & $0.922 \pm 0.053$ & $3.88 / 8$ & $0.882 \pm 0.083$ & $6.07 / 7$ & $0.911 \pm 0.045$ & $10.11 / 16$ \\
4 & $0.914 \pm 0.056$ & $10.14 / 8$ & $0.790 \pm 0.046$ & $20.79 / 14$ & $0.840 \pm 0.036$ & $33.82 / 23$ \\
5 & $0.868 \pm 0.049$ & $14.46 / 10$ & $0.677 \pm 0.045$ & $14.02 / 12$ & $0.764 \pm 0.033$ & $36.65 / 23$ \\
6 & $0.869 \pm 0.055$ & $10.32 / 6$ & $0.784 \pm 0.070$ & $13.98 / 7$ & $0.837 \pm 0.043$ & $25.23 / 14$ \\
7 & $0.907 \pm 0.066$ & $2.50 / 6$ & $0.638 \pm 0.062$ & $9.84 / 8$ & $0.764 \pm 0.045$ & $21.16 / 15$ \\
$\sim 9$ & $0.837 \pm 0.052$ & $5.92 / 8$ & $0.703 \pm 0.107$ & $9.18 / 3$ & $0.812 \pm 0.047$ & $16.37 / 12$ \\
\hline
\end{tabular}



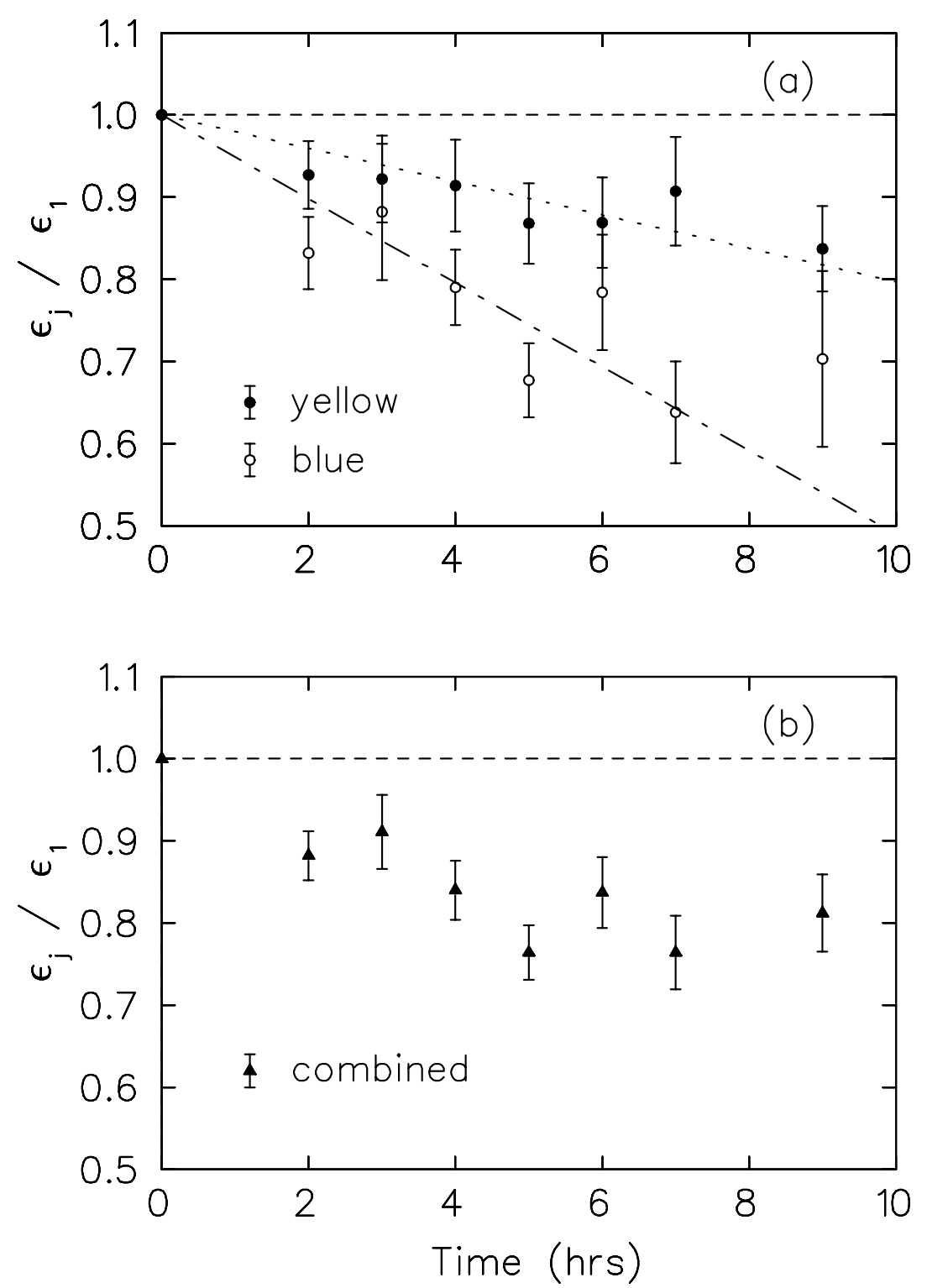

FIG. 23: Asymmetry ratios as a function of time from the beginning of flattop. (a) Individual results for the Blue and Yellow ring. (b) Combined data from the two rings.

1.00 and decrease with time. A linear fit was done to data from the Yellow ring forcing the fit to go through the first point. This gave a slope of $-.0203 \pm .0037$. For the Blue ring a similar fit gives a slope of $-.0511 \pm .0045$. This can be interpreted as a depolarization rate of $2 \%$ per hour and $5 \%$ per hour for the Yellow and Blue ring, respectively, or of decay times of 49 and 20 hours. Alternately, one might hypothesize that the RHIC polarimeter had systematic errors which are correlated with the changing beam conditions during the 
store.

An obvious interpretation is that the RHIC beams were depolarizing on flattop. However, it is also possible that changes in beam phase space or systematic effects in the polarimeters, possibly correlated with the lower rates later in the fills, contributed to the observed drop in measured asymmetries.

\section{SUMMARY}

The following conclusions can be drawn from the analyses in this note:

- Recalibration measurements were performed for the $200-\mathrm{MeV}$ polarimeter using the $p+d$ elastic scattering reaction. The results are consistent with the older calibration for the $12^{\circ}$ arms.

- High rates in the $200-\mathrm{MeV}$ polarimeter due to the higher beam currents from the new optically pumped polarized ion source are still a problem, even after some hardware changes. Further improvements to make both the $p+C$ and $p+d$ systems more rate tolerant are required. The $p+C$ calibration should be repeated after these improvements.

- The AGS (E880) polarimeter showed no direct evidence of systematic effects comparable to statistical uncertainties in RHIC run 2. There was also no evidence for systematic effects on the basis of new diagnostics or tests (see Figs. 3, 5, 6). However, higher counting rates in the E880 polarimeter scintillation counters in the past have produced significant systematic errors.

- It appears that the beam polarization was similar at $200 \mathrm{MeV}$ and at $G \gamma=7.5(3.8$ $\mathrm{GeV} / \mathrm{c}$ ) during the RHIC run 2 period. However, there was apparently time-dependent depolarization between $G \gamma=7.5$ and 12.5 , probably caused by the $G \gamma=0+\nu$ intrinsic resonance, and at higher energies.

- A comparison of luminosity asymmetries for the three pairs of detectors in each RHIC CNI polarimeter indicated large numbers of statistically significant differences throughout the run period. The origin of these differences is not presently understood, but the problems appear worse in the 1-4 detector pair in the Blue and the 2-5 detector 
pair in the Yellow ring polarimeter. There are also significant numbers of runs with nonzero up-down and cross asymmetries, $\epsilon_{Y 45}$ and $\epsilon_{c r 45}$, which are not expected. It appears $\epsilon_{Y 45}$ is somewhat correlated with the luminosity asymmetry differences. These observations suggest that there may be sizable systematic effects in the physics asymmetries, so that a $<5 \%$ measurement of beam polarization may not be possible under the present conditions.

- An estimate of the effect of vertical beam position correlated with beam spin direction suggested this was not the cause of the large differences in luminosity asymmetries or of the nonzero cross and up-down physics asymmetries.

- A search for correlations between the X90 physics asymmetry, $\epsilon_{X 90}$, and the associated luminosity asymmetry was unsuccessful. However, a weak correlation was observed between $\epsilon_{X 90}$ and the X90 geometrical asymmetry, but RHIC operating conditions may have changed over the four days of the data set tested causing the observed correlation.

- The mean ratio of RHIC polarimeter asymmetries at injection for the two rings was found to be consistent with the ratio of analyzing powers for $\epsilon_{X 45}$ and the least squares fit $\mathrm{X}$ asymmetry. The ratio for $\epsilon_{X 90}$ differed by about four standard deviations from the other two ratios. Fluctuations in the ratios are outside statistics, but comparable to inferred systematics effects in luminosity asymmetry differences, as well as $\epsilon_{Y 45}$ and $\epsilon_{c r 45}$.

- The ratio of flattop to injection analyzing powers for the RHIC polarimeter is $A_{\text {flat }} / A_{i n j} \geq 1.0$, based on the observed ratio of asymmetries. Using this condition, there were many RHIC fills with significant polarization losses during acceleration to flattop. This is consistent with the sizable differences in flattop asymmetries in the two polarimeters that were frequently observed.

- On average, the magnitude of the flattop asymmetry decreased over the course of a fill. The rate of decrease in the Blue ring ( $\sim 5 \%$ per hour) was higher than in the Yellow ring.

Based on the last two conclusions, it appears that in future runs time should be devoted to minimize polarization losses during acceleration and flattop in RHIC. 


\section{ACKNOWLEDGEMENTS}

We wish to thank the AGS staff for their support and help with the measurements of the polarimeter data. This research was supported in part by the U.S. Department of Energy, Division of High Energy Physics, Grant No. DE-FG02-92ER40747 and Contract No. W-31109-ENG-38, the U.S. National Science Foundation, and the Science and Technology Agency of Japan.

[1] O. Jinnouchi et al., AIP Conference Proceedings 675, 817 (2003); O. Jinnouchi in "Proceedings of RIKEN BNL Research Center Workshop", BNL reports BNL-71117-2003 (2003), p57, 141, 311, and BNL-71118-2003 (2003), p93, and BNL-71300-2003 (2003), p49.

[2] E.J. Stephenson et al., in Proceedings of the RHIC Spin Collaboration Meeting VI, Brookhaven National Laboratory report BNL-52642, p64-69 (2001).

[3] F.Z. Khiari et al., Phys. Rev. D 39, 45 (1989).

[4] J.B. Roberts et al., IUCF Sci. and Tech. Report - 1982, p. 166; see also p. 201.

[5] S.P. Wells et al., Nucl. Instrum. Methods A325, 205 (1993).

[6] E.J. Stephenson, in Applications of Accelerators in Research and Industry (AIP Conf. Proc. 576, New York, 2001) p. 575.

[7] M.W. McNaughton et al., Nucl. Instrum. Methods A241, 435 (1985).

[8] H. Huang, R.V. Cadman, H. Spinka, and D. Underwood, Brookhaven National Laboratory technical note C-A/AP/93 (2003, unpublished).

[9] W.W. MacKay and N. Tsoupas, AIP Conference Proceedings 667, 84 (2003); and W. MacKay, private communication. 